\title{
THE
}

\section{On the Equilibrium-State Roll Vortices and Their Effects in the Hurricane Boundary Layer}

\author{
Kun Gao \\ University of Rhode Island, kun_gao@my.uri.edu \\ Isaac Ginis \\ University of Rhode Island, iginis@uri.edu
}

Follow this and additional works at: https://digitalcommons.uri.edu/gsofacpubs

Creative Commons License

\section{(c) (i)}

This work is licensed under a Creative Commons Attribution 4.0 License.

\section{Citation/Publisher Attribution}

Gao, K. and Ginis, I. (2016). On the equilibrium-state roll vortices and their effects in the hurricane boundary layer. Journal of the Atmospheric Sciences 73(3): 1205-1222. doi: 10.1175/JAS-D-15-0089.1 Available at: http://dx.doi.org/10.1175/JAS-D-15-0089.1

This Article is brought to you for free and open access by the Graduate School of Oceanography at DigitalCommons@URI. It has been accepted for inclusion in Graduate School of Oceanography Faculty Publications by an authorized administrator of DigitalCommons@URI. For more information, please contact digitalcommons-group@uri.edu. 


\title{
¿On the Equilibrium-State Roll Vortices and Their Effects in the Hurricane Boundary Layer
}

\author{
KUN GAO AND ISAAC GINIS \\ Graduate School of Oceanography, University of Rhode Island, Narragansett, Rhode Island
}

(Manuscript received 20 March 2015, in final form 6 October 2015)

\begin{abstract}
In this study, the authors numerically simulate roll vortices (rolls) generated by the inflection-point instability in the hurricane boundary layer (HBL). The approach is based on embedding a two-dimensional high-resolution single-grid roll-resolving model (SRM) at selected horizontal grid points of an axisymmetric HBL model. The results from a set of idealized experiments indicate that the mixed-layer height is an important factor affecting the magnitude of the roll velocities and the structure of the internal waves triggered in the stably stratified layer above. This study reveals the important difference between the roll-induced cross-roll (nearly radial) and alongroll (nearly azimuthal) momentum fluxes: while the cross-roll momentum flux is well correlated to the cross-roll mean wind shear, the along-roll momentum flux is typically not correlated with the along-roll mean wind shear. Therefore, the commonly used $K$ theory in the boundary layer parameterizations cannot reasonably capture the vertical distribution of the roll-induced along-roll momentum flux. Moreover, the authors find that the rolls induce more significant changes in the mean radial wind profile than in the mean azimuthal wind profile. Specifically, rolls reduce the inflow near surface, enhance the inflow at upper levels, and increase the inflow-layer height. Based on a linear dynamical HBL model, the authors find that the impact of rolls on the mean radial wind profile is essentially due to their redistribution effect on the mean azimuthal momentum in the HBL.
\end{abstract}

\section{Introduction}

Roll vortices (herein "rolls") refer to the coherent counterrotating vortices in the atmospheric boundary layer, which are approximately aligned in the mean wind direction (Etling and Brown 1993). Rolls have been reported to frequently occur in the hurricane boundary layer (HBL) in observational studies (Wurman and Winslow 1998; Morrison et al. 2005; Lorsolo et al. 2008; Zhang et al. 2008; Ellis and Businger 2010). Most of the observations were made by Doppler radars during landfalling hurricanes and helped to quantify some characteristics of rolls. For example, Morrison et al. (2005) estimated the average horizontal wavelength of $\sim 1.5 \mathrm{~km}$, the average depth of $\sim 700 \mathrm{~m}$, the average vertical velocity of $\sim 3 \mathrm{~m} \mathrm{~s}^{-1}$, and the average horizontal

¿ Denotes Open Access content.

Corresponding author address: Kun Gao, Graduate School of Oceanography, University of Rhode Island, 215 South Ferry Rd., Narragansett, RI 02882.

E-mail: kun_gao@my.uri.edu wind speed perturbations of $\sim 7 \mathrm{~m} \mathrm{~s}^{-1}$. Zhang et al. (2008) reported the first in situ measurements of the rollinduced vertical fluxes and showed that the rolls significantly enhanced the total momentum and moisture transports in the HBL. Because of the large vertical extent and strong vertical motions of rolls, the nonlocal effects induced by rolls may not be adequately represented by the existing turbulence parameterizations in hurricane models (Foster 2005). That is possibly an important factor limiting the current hurricane model forecast skill.

The formation mechanism and characteristics of rolls in the classical Ekman boundary layer have been extensively studied (e.g., Faller 1965; Lilly 1966; Brown 1970, 1972; Etling and Raasch 1987) and summarized in the literature (e.g., Brown 1980; Etling and Brown 1993). Thus we only review here the previous theoretical and numerical studies that focused on rolls in the HBL. Foster (2005) applied stability analyses to the basic-state HBL flow and demonstrated that the inflection points in the radial wind profiles caused the instability and led to the formation of rolls. This generation mechanism is often referred to as the inflection point instability. In a more recent study, Foster (2013) found that rolls with wavelength $O(10) \mathrm{km}$ could be formed by the nonlinear 
wave-wave interaction. Nolan (2005) used both a stability analysis approach and a nonlinear axisymmetric model and also found that inflection points in the HBL wind profiles were responsible for the formation of rolls. Subsequent numerical studies based on large-eddy simulation (LES) concurred that rolls in the HBL can be generated by the inflection point instability. Zhu (2008) nested an LES model within the WRF regional model to simulate a landfalling hurricane. He found rolllike perturbations in a statically stable boundary layer, which were likely generated by the dynamical instability rather than the convective instability. He also pointed out that the current boundary layer schemes underestimated the turbulent fluxes in the HBL because the effects of coherent large eddies were not effectively included. Nakanishi and Niino (2012) used an LES model for the idealized HBL and identified rolls generated by the inflection point instability based on the EOF analysis of the LES results. They also suggested that rolls could interact with other small-scale features (such as internal waves and Kelvin-Helmholtz waves), and the rolls enhanced the vertical transports in the HBL. In a recent study by the authors (Gao and Ginis 2014), rolls were resolved by applying a two-dimensional high-resolution single-grid roll-resolving model (SRM) embedded into an axisymmetric HBL model. They found that rolls generated by the inflection point instability are affected by the stratification during the linear phase, in which the rolls grow exponentially with time. Particularly, the mixed layer height affects the growth rate of rolls and the structure of the internal waves in the stably stratified layer, which are triggered by the rolls.

While the previous studies advanced the understanding of the formation mechanism and characteristics of rolls in the HBL, some important aspects of rolls in the HBL still remain unexplored. As indicated by previous studies (Zhu 2008; Nakanishi and Niino 2012), the rolls can significantly contribute to the vertical transports in HBL. However, the characteristic distributions of the roll-induced vertical momentum fluxes and their correlations with the vertical gradients of the mean flow have not been studied. Moreover, there is still limited understanding of the impacts of rolls on the mean HBL flow. In this study, we aim to advance the understanding on these important aspects of rolls in the HBL by extending the numerical approach used by Gao and Ginis (2014). Specifically, we address the following questions:

(i) How does the mixed-layer height affect the rolls in the nonlinear phase and the internal waves that are triggered in the stably stratified layer above?

(ii) Can the roll-induced momentum fluxes be reasonably represented by the commonly used $K$ theory? (iii) What are the effects of rolls on the mean wind distribution in the HBL and what is the underlying physical mechanism?

\section{Method}

\section{a. Modeling approach}

Our modeling approach is based on the assumption that rolls can be separated from the large-scale flow because of their small spatial scale. The total resolvable flow in the HBL is split into two components: the mean flow and the perturbations. Taking velocity $\mathbf{v}$ as an example, we assume $\mathbf{v}=\overline{\mathbf{v}}+\mathbf{v}^{\prime}$, where $\overline{\mathbf{v}}$ represents the mean wind and $\mathbf{v}^{\prime}$ represents the wind perturbations. In this study, the mean flow refers to the large-scale flow in the HBL, which consists of the primary cyclonic circulation and the secondary circulation induced by the surface friction; the perturbations refer to the smallscale features formed as a result of the dynamical instability of the mean flow, which consist of the boundary layer rolls and the internal waves triggered by them. Based on the above flow-separation assumption, the governing equations for the mean flow and the perturbations can be derived, as shown in the appendix of Gao and Ginis (2014). The equations for the mean flow and the perturbations are solved by two different numerical models, while the unresolved small-scale turbulence are parameterized using the traditional method.

Two different coordinate systems are used to describe the mean flow and the perturbations. The HBL mean flow is assumed axisymmetric and therefore can be described in a cylindrical coordinate system $(r, \lambda, z)$. For the perturbations, similar to Foster (2005), we use a local Cartesian coordinate system $(x, y, z)^{1}$ (Fig. 1$)$. The system $(x, y, z)$ is set up in such a way that the $y$ axis is parallel to the direction along which the rolls are aligned. We will refer to the $y(x)$ axis as the along-roll (cross roll) axis. The along-roll variations of the perturbations are assumed negligible; that is, $\partial a^{\prime} / \partial y^{\prime}=0$, where $a^{\prime}$ represents the perturbations. Note the twodimensional assumption filters out three-dimensional large eddies that may also exist in the HBL. However, since this study primarily focuses on the quasi-twodimensional rolls, it is reasonable to apply this assumption. To distinguish the wind components projected onto

\footnotetext{
${ }^{1}$ The approximation of using the local Cartesian coordinates instead of the cylindrical coordinates is only valid at relatively large radii where the curvature $(1 / r)$ is sufficiently small. In this study, we only consider perturbations at and outside of the radius of maximum wind (see section $2 \mathrm{c}$ for the reason) and we assume such approximation is reasonable at the radii we consider.
} 


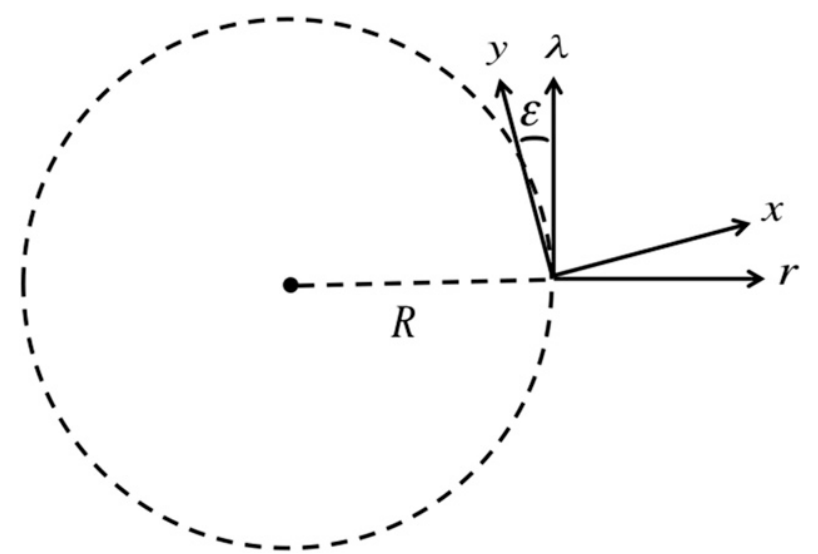

FIG. 1. A diagram illustrating the cylindrical coordinate system $(r, \lambda, z)$ for the mean flow and the local Cartesian coordinate system $(x, y, z)$ for the perturbations ( $z$ axis is not shown) at $r=R$ [as in Fig. 1 of Gao and Ginis (2014)]. The variable $\varepsilon$ is the angle between the along-roll direction $y$ and the azimuthal direction $\lambda$. Positive $\varepsilon$ means the along-roll direction is to the left of the azimuthal direction.

the two different coordinate systems, uppercase letters $(U, V, W)$ will represent the wind components in the cylindrical coordinates and lowercase letters $(u, v, w)$ will represent the wind components in the local Cartesian coordinate system. If the angle between the alongroll direction $y$ and the azimuthal direction $\lambda$ is defined as $\varepsilon$ (Fig. 1), the wind components in the two coordinate systems can be transformed as follows:

$$
\left(\begin{array}{l}
u \\
v
\end{array}\right)=\left(\begin{array}{cc}
\cos \varepsilon & \sin \varepsilon \\
-\sin \varepsilon & \cos \varepsilon
\end{array}\right)\left(\begin{array}{l}
U \\
V
\end{array}\right) \text { and } w=W
$$

\section{b. The HBL model}

The governing equations for the mean wind components in the HBL are

$$
\begin{aligned}
& \frac{\partial \bar{U}}{\partial t}+\bar{U} \frac{\partial \bar{U}}{\partial r}+\bar{W} \frac{\partial \bar{U}}{\partial z}-\frac{\bar{V}^{2}}{r}-f \bar{V} \\
& =-\frac{1}{\rho_{0}} \frac{\partial \bar{P}}{\partial r}+\frac{\partial}{\partial z}\left(\bar{K} \frac{\partial \bar{U}}{\partial z}\right)-\frac{\partial \overline{W^{\prime} U^{\prime}}}{\partial z}, \\
& \frac{\partial \bar{V}}{\partial t}+\bar{U} \frac{\partial \bar{V}}{\partial r}+\bar{W} \frac{\partial \bar{V}}{\partial z}+\frac{\bar{U} \bar{V}}{r}+f \bar{U} \\
& =\frac{\partial}{\partial z}\left(\bar{K} \frac{\partial \bar{V}}{\partial z}\right)-\frac{\partial \overline{W^{\prime} V^{\prime}}}{\partial z}, \text { and } \\
& \frac{\partial \bar{U}}{\partial r}+\frac{\bar{U}}{r}+\frac{\partial \bar{W}}{\partial z}=0,
\end{aligned}
$$

where $\bar{U}, \bar{V}$, and $\bar{W}$ are the mean wind components in the radial, azimuthal, and vertical directions, respectively; $\overline{W^{\prime} U^{\prime}}$ and $\overline{W^{\prime} V^{\prime}}$ are the radial and azimuthal

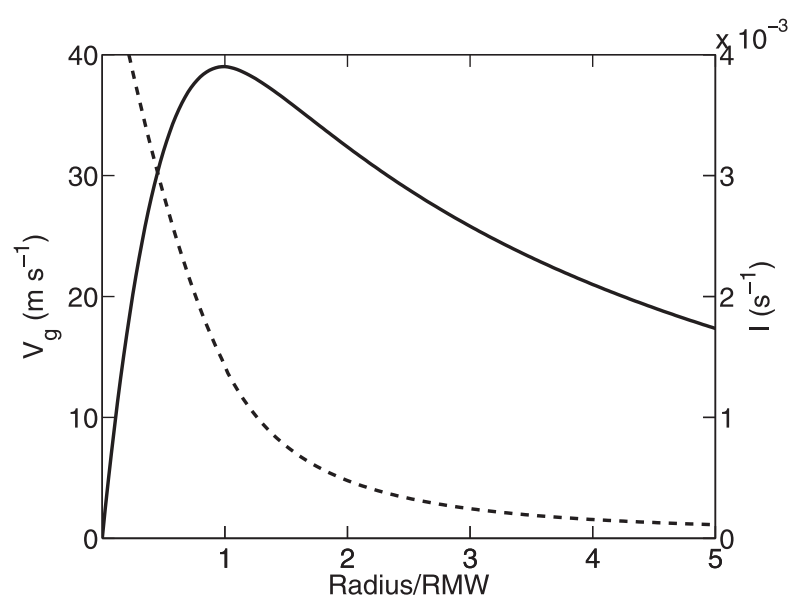

FIG. 2. The gradient wind (solid line; left $y$ axis) and inertial stability parameter (dashed line; right $y$ axis) as functions of radius normalized by the radius of maximum wind [as in Fig. 2 of Gao and Ginis (2014)].

momentum fluxes induced by the perturbations, respectively; $\bar{K}$ is the parameterized turbulent diffusivity (the parameterization of $K$ is discussed in section $2 \mathrm{~d}$ ). Similar equations are used to describe the HBL mean wind in other studies (e.g., Foster 2009; Kepert 2012). A major difference here is that we explicitly consider the momentum tendencies induced by the resolved perturbations in (2) and (3).

The numerical model solving (2)-(4) is hereafter referred to as the HBL model. At the upper boundary $(z=$ $H$, where $H$ is the vertical extent of the atmospheric layer in the HBL model and is set to $3 \mathrm{~km}$ ), we assume that the mean wind is under the gradient wind balance; that is, $\bar{V}=V_{g}$ and $\bar{U}=0$, where $V_{g}$ is the gradient wind, satisfying

$$
-\frac{V_{g}^{2}}{r}-f V_{g}=-\frac{1}{\rho_{0}} \frac{\partial \bar{P}}{\partial r},
$$

where $\rho_{0}$ is a constant. The radial distribution of $V_{g}$ is prescribed, and the pressure gradient force derived from (5) is assumed vertically uniform. The Holland (1980) parametric model is used to specify the radial distribution of $V_{g}$. The hurricane is assumed to be on an $f$ plane at $20^{\circ} \mathrm{N}$ with maximum $V_{g}$ of $39 \mathrm{~m} \mathrm{~s}^{-1}$, the radius of maximum wind (RMW) of $40 \mathrm{~km}$, and the parameter $B$ of 1.3 ( $B$ controls how rapidly $V_{g}$ decreases with radius larger than the RMW). The value of $V_{g}$ inside of the RMW is specified following the formulation in Kepert and Wang (2001). Figure 2 shows the radial distribution of $V_{g}$ and the inertial stability parameter given by $I=\sqrt{\left(f+2 V_{g} / r\right)\left(f+V_{g} / r+\partial V_{g} / \partial r\right)} \quad$ (Kepert 2001). The surface layer is parameterized using the MoninObukhov similarity theory with the roughness length 
formulation proposed by Moon et al. (2007), which limits the drag coefficient below 0.003 at high wind speeds.

Another important variable is the mean virtual potential temperature $\bar{\theta}_{v}$. Since the idealized HBL model used in this study cannot fully capture the physical processes that determine $\bar{\theta}_{v}$, its vertical profile is specified using the analytical formula described in section 3 .

\section{c. The single-grid roll-resolving model}

The governing equations describing the perturbations at a horizontal grid point in the HBL model are

$$
\begin{aligned}
\frac{\partial \eta^{\prime}}{\partial t}+u^{\prime} \frac{\partial \eta^{\prime}}{\partial x}+w^{\prime} \frac{\partial \eta^{\prime}}{\partial z} & =-\bar{u} \frac{\partial \eta^{\prime}}{\partial x}+w^{\prime} \frac{\partial^{2} \bar{u}}{\partial z^{2}}+\frac{g}{\theta_{v 0}} \frac{\partial \theta_{v}^{\prime}}{\partial x}+T_{\eta^{\prime}} \\
\frac{\eta^{\prime}}{\partial v^{\prime}}+u^{\prime} \frac{\partial v^{\prime}}{\partial x}+w^{\prime} \frac{\partial \psi^{\prime}}{\partial z} & =-\bar{u} \frac{\partial^{2} \psi^{\prime}}{\partial z^{2}} \\
\frac{\partial \theta_{v}^{\prime}}{\partial t}+u^{\prime} \frac{\partial \theta_{v}^{\prime}}{\partial x}+w^{\prime} \frac{\partial \theta_{v}^{\prime}}{\partial z} & =-\bar{u} \frac{\partial \theta_{v}^{\prime}}{\partial x}-w^{\prime} \frac{\partial \bar{\theta}_{v}}{\partial z}+T_{\theta_{v_{v}^{\prime}}}
\end{aligned}
$$

Variables with the prime sign are associated with the perturbations: $\left(u^{\prime}, v^{\prime}, w^{\prime}\right)$ are the velocity components in the local Cartesian coordinates, $\psi^{\prime}$ is the streamfunction $\left(u^{\prime}=-\partial \psi^{\prime} / \partial z, w^{\prime}=\partial \psi^{\prime} / \partial x\right), \eta^{\prime}$ is the along-roll vorticity $\left(\eta^{\prime}=\partial w^{\prime} / \partial x-\partial u^{\prime} / \partial z\right)$, and $\theta_{v}^{\prime}$ is the virtual potential temperature perturbation. Variables with the overbar are associated with the mean flow: $\bar{u}$ and $\bar{v}$ are the mean winds projected onto the cross-roll $(x)$ and along-roll $(y)$ directions, respectively, and they are provided by the HBL model. In (6) $\theta_{v 0}$ is a constant and is set to $300 \mathrm{~K}$. The variables $T_{\eta^{\prime}}, T_{v^{\prime}}$, and $T_{\theta_{v}^{\prime}}$ represent the turbulent diffusions; take $T_{\theta_{v}^{\prime}}$ for example-it is in the form $T_{\theta_{v}^{\prime}}=\partial\left(K \partial \theta_{v}^{\prime} / \partial x\right) / \partial x+\partial\left(K \partial \theta_{v}^{\prime} / \partial z\right) / \partial z$, where $K$ is the turbulent diffusivity (section $2 \mathrm{~d}$ ). The numerical model resolving (6)-(9) is herein referred to as the SRM. Its function is to resolve the perturbations at a single horizontal grid point of the HBL model. In the above equations for perturbations, the mean-flow variables are assumed horizontally uniform within the SRM domain. Such assumption may not be valid at the locations within the RMW where the wind speed increases rapidly with radius. Therefore, we only consider the perturbations at and outside of the RMW in this study. The Coriolis and centrifugal terms in (6) and (8) are neglected because we find that these terms do not affect the solutions in any significant way at the radii we consider, which is consistent with Foster (2005). As for the boundary conditions, no-slip condition is applied at the upper and lower boundaries of the SRM domain (that is $u^{\prime}=v^{\prime}=w^{\prime}=\theta_{v}^{\prime}=0$ ), and the periodic condition is applied at the lateral boundaries.

\section{d. Turbulent diffusivity parameterization}

For simplicity, the turbulent diffusivity for momentum and heat are assumed the same in this study. The turbulent diffusivity is parameterized by a first-order scheme as in Zhang and Drennan (2012) and Kepert (2012). In this scheme, $K$ is given in the form of $K=l^{2} S f(\mathrm{Ri})$, where $l$ is the mixing length, $S$ is the strain rate, and $\mathrm{Ri}$ is the gradient Richardson number.

(i) The mixing length $l$ has the form suggested by Blackadar (1962), $l^{-1}=(\kappa z)^{-1}+l_{\infty}^{-1}$, where $\kappa$ is the von Kármán constant $(\kappa=0.4)$ and $l_{\infty}$ is asymptotic mixing length, ${ }^{2}$ which is set to $30 \mathrm{~m}$. The impacts of the choice of $l_{\infty}$ on the formation and characteristics of rolls were discussed in Gao and Ginis (2014). Here a relatively small value of $l_{\infty}$ is used to ensure the mean flow is favorable for rolls to form.

(ii) The strain rate $S$ is given by $S^{2}=1 / 2\left(\partial u_{i} / \partial x_{j}+\right.$ $\left.\partial u_{j} / \partial x_{i}\right)^{2}$, where $u_{i}=\bar{u}_{i}+u_{i}^{\prime}$.

(iii) The function $f(\mathrm{Ri})=\sqrt{1-\mathrm{Ri}}$ and $\mathrm{Ri}$ is defined as $\mathrm{Ri}=g \theta_{v 0}^{-1} S^{-2} \partial \theta_{v} / \partial z$, where $\theta_{v}=\bar{\theta}_{v}+\theta_{v}^{\prime}$.

The calculations of $K$ at a horizontal grid point of the HBL model are as follows. If SRM is not embedded, $\bar{K}$ in the mean wind equations (2) and (3) are calculated with $S$ equal to the mean wind shear, given by $S^{2}=(\partial \bar{U} / \partial z)^{2}+(\partial \bar{V} / \partial z)^{2}$. If SRM is embedded, the total turbulent diffusivity $K$ in roll equations (6), (8), and (9) are calculated with $S$ given by $S^{2}=2(\partial u / \partial x)^{2}+2(\partial w / \partial z)^{2}+(\partial u / \partial z+\partial w / \partial x)^{2}+$ $(\partial v / \partial x)^{2}+(\partial v / \partial z)^{2}$, where the wind components are the total resolved winds projected onto the local Cartesian coordinates-for example, $u=\bar{u}(z)+u^{\prime}(x, z)$; the horizontally averaged $K$ in the SRM domain, that is $\bar{K}$, is applied in (2) and (3).

\section{e. The HBL-SRM coupled system}

The HBL model and the SRM are dynamically coupled and integrated in time simultaneously. The SRM can be embedded into the HBL model at multiple horizontal grid points. The HBL model provides the mean wind profiles to the SRM at selected grid points, and SRM provides the HBL model with the vertical

\footnotetext{
${ }^{2}$ The asymptotic mixing length $l_{\infty}$ is an important factor affecting the mean HBL wind distribution because it directly affects the magnitude of the turbulent diffusivity $K$. The characteristics of rolls are sensitive to the choice of $l_{\infty}$ because they are generated as a result of the instability of the mean radial wind distribution in the HBL [not shown in this study; please see Gao and Ginis (2014) for details]. Nevertheless, the vertical distribution of the roll-induced momentum fluxes and their impacts on the mean wind profiles, which are the main focuses of this study, are not qualitatively affected by the choice of $l_{\infty}$.
} 
TABLE 1. Numerical parameters for the HBL model and the SRM.

\begin{tabular}{lcc}
\hline & HBL model & SRM \\
\hline Vertical extent & $3 \mathrm{~km}$ & $6 \mathrm{~km}$ \\
Horizontal extent & $1000 \mathrm{~km}$ & $15.36 \mathrm{~km}$ \\
Vertical grid spacing & $30 \mathrm{~m}$ & $30 \mathrm{~m}$ \\
Horizontal grid spacing & $10 \mathrm{~km}$ & $30 \mathrm{~m}$ \\
Time step & $1 \mathrm{~s}$ & $1 \mathrm{~s}$ \\
\hline
\end{tabular}

momentum fluxes induced by the resolved perturbations. The numerical parameters for the HBL model and the SRM are listed in Table 1. Note that the vertical extent of the SRM domain $(6 \mathrm{~km})$ is set to be higher than the vertical extent of the HBL model domain $(3 \mathrm{~km})$. The Rayleigh damping is applied in the upper $3 \mathrm{~km}$ of the SRM domain to suppress the vertically propagating internal waves and prevent them from affecting the boundary layer solutions.

In each numerical experiment, the HBL model is run first for $24 \mathrm{~h}$ without the effects of perturbations to derive a steady-state mean wind distribution. Then the SRM is embedded at selected grid points in the HBL model and the two models are run in the coupled mode. An infinitesimal perturbation in the along-roll vorticity field $\eta^{\prime}$ is introduced as the initial condition for the perturbations.

\section{Experimental design}

The primary set of experiments, hereafter M200M600, are designed with two purposes: (i) to investigate how the stratification, particularly the mixed layer height, affects the characteristics of the perturbations and (ii) to reveal the impacts of rolls at a particular radius on the local mean wind profiles. In these experiments the SRM is only embedded at the RMW in the HBL model. The initial mean wind profiles are kept the same, ${ }^{3}$ but the initial $\bar{\theta}_{v}$ profiles are varied and prescribed analytically based on the composite GPS dropsondes measurements described in Zhang et al. (2011). The formula for the vertical gradient of $\bar{\theta}_{v}$ is given by

$$
\frac{\partial \bar{\theta}_{v}}{\partial z}=A \tanh ^{2}\left(\frac{z}{h}\right)
$$

where $A$ is the background value for $\partial \bar{\theta}_{v} / \partial z$ and $h$ is a depth scale controlling the mixed-layer height. The vertical profile of $\bar{\theta}_{v}$ can be derived by integrating $\partial \bar{\theta}_{v} / \partial z$ vertically. Figure 3 shows a typical analytical profile of

\footnotetext{
${ }^{3}$ The initial $\bar{\theta}_{v}$ profiles can affect the initial mean wind profiles through affecting the static stability factor in the $K$ parameterization. However, it is found that such influence is very weak.
}

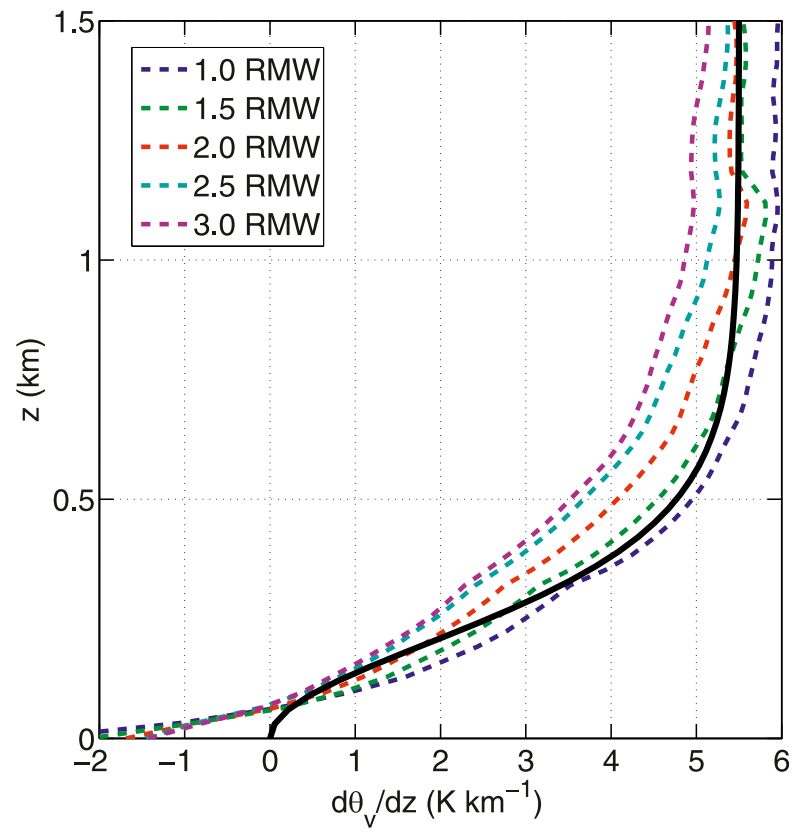

FIG. 3. Vertical profiles of the vertical gradient of the virtual potential temperature in the HBL. Dashed lines are typical profiles from the composite observational dataset (Zhang et al. 2011) and the locations of these profiles are from 1RMW to 3RMW with a $0.5 \mathrm{RMW}$ interval. The black solid line is the profile specified based on (10) in the text, with $A=5.5 \mathrm{~K} \mathrm{~km}^{-1}$ and $h=300 \mathrm{~m}$.

$\partial \bar{\theta}_{v} / \partial z$, as well as several observed profiles from Zhang et al. (2011) for comparison. The analytical $\partial \bar{\theta}_{v} / \partial z$ profile gradually increases from $0 \mathrm{~K} \mathrm{~km}^{-1}$ to the background value $A=5.5 \mathrm{~K} \mathrm{~km}^{-1}$, similar to the observed profiles. ${ }^{4}$ Figure 4 shows the vertical profiles of $\bar{\theta}_{v}$ and $\partial \bar{\theta}_{v} / \partial z$ in the primary set of experiments. The mixed-layer height, defined as the height where $\partial \bar{\theta}_{v} / \partial z$ is equal to $3 \mathrm{~K} \mathrm{~km}^{-1}$ (Zhang et al. 2011), varies from 200 to $600 \mathrm{~m}$, with an increment of $100 \mathrm{~m}$. These prescribed $\bar{\theta}_{v}$ profiles are held unchanged with time. Figure 5 shows the initial mean wind profiles at the RMW (radius $=40 \mathrm{~km}$ ) in the primary set of experiments, which are derived by running the HBL model for $24 \mathrm{~h}$ without considering the momentum tendencies induced by the perturbations.

\footnotetext{
${ }^{4}$ The observed $\bar{\theta}_{v}$ profiles (Fig. 3) suggest that a shallow unstably stratified layer $\left(\partial \bar{\theta}_{v} / \partial z<0\right)$ exists near the surface $(z<100 \mathrm{~m})$. To test the effect of the unstably stratified layer on the characteristics of the rolls, we conducted a series of sensitivity experiments. The height of the unstably stratified layer and the lapse rate within this layer were constrained by the observed values (Zhang et al. 2011). The effects on the characteristics of rolls, including their basic structures, kinetic energy, and fluxes, were found to be insignificant. Thus for the sake of simplicity, we chose to initialize all the experiments presented in this paper without the unstably stratified layer.
} 


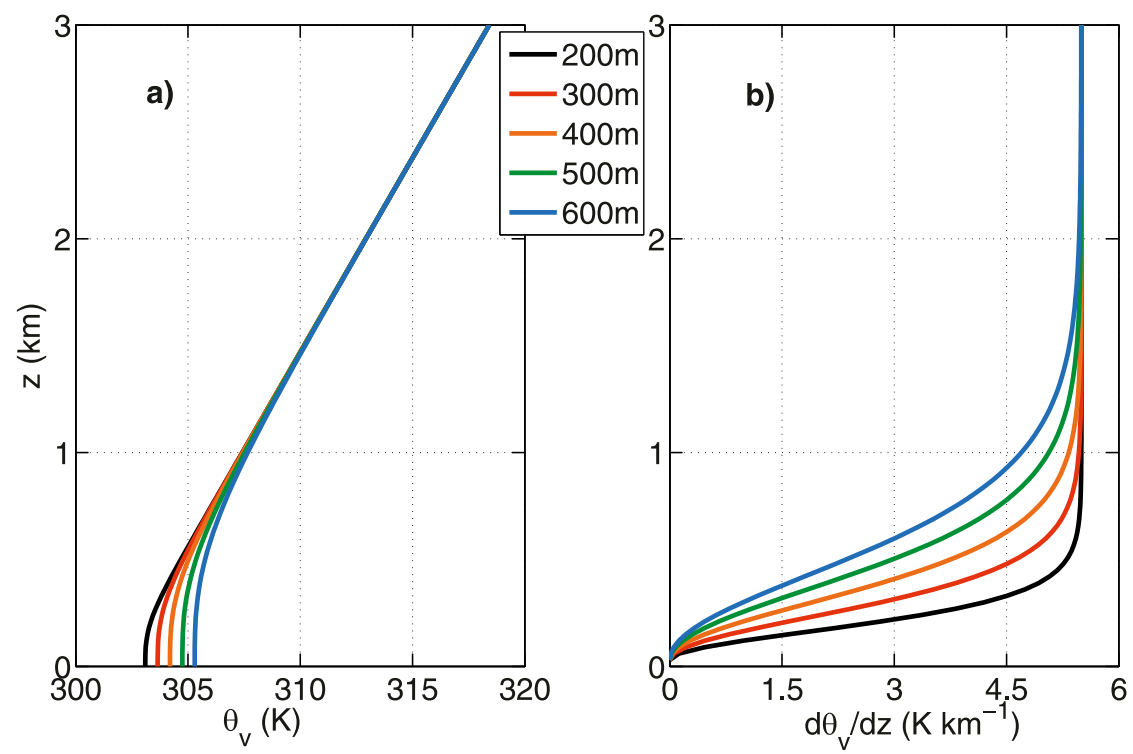

FIG. 4. Vertical profiles of (a) the initial mean virtual potential temperature and (b) its vertical gradient used in the primary set of experiments M200-M600. The heights of the mixed layer in these profiles are indicated in the legend.

In an additional experiment, SRM is embedded at all horizontal grid points (except those inside of the RMW) in the HBL model to investigate the impacts of the rolls on the overall mean wind distribution in the HBL. In this experiment, the same $\bar{\theta}_{v}$ profile is used at all locations with the mixed-layer height set to $600 \mathrm{~m}$. A relatively high mixed layer is used to ensure that rolls can reach maximum magnitude if they are generated.

In all experiments the angle $\varepsilon$ (Fig. 1) between the $y$ axis and the $\lambda$ axis is calculated based on the mean wind

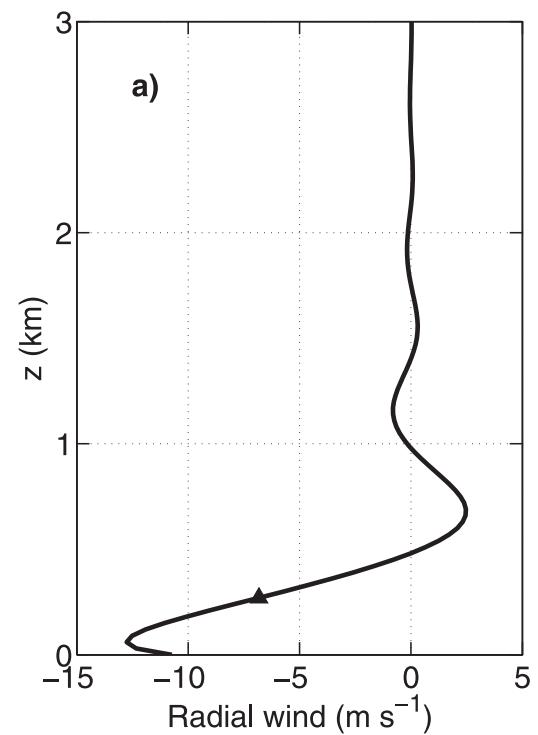

profiles in the HBL. Previous observations and theoretical studies (Morrison et al. 2005; Foster 2005) suggest that rolls tend to align in the direction of the depth-averaged wind. Here we assume that the along-roll axis ( $y$ axis) is in the direction of the depthaveraged wind vector below $1 \mathrm{~km}$. The calculated angle $\varepsilon$ varies with distance from $\sim 4^{\circ}$ (at RMW) to $\sim 10^{\circ}$ (at 3 RMW), which is consistent with Foster (2005) (please see appendix B for the sensitivity of the model results to $\varepsilon$ ).

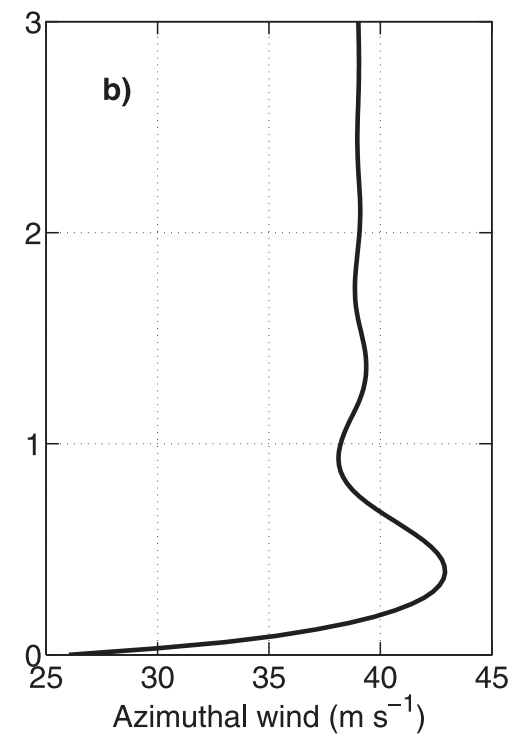

FIG. 5. Vertical profiles of the initial mean (a) radial wind and (b) azimuthal wind at the RMW. The inflection point with the largest radial wind shear is indicated by the triangle in (a). 


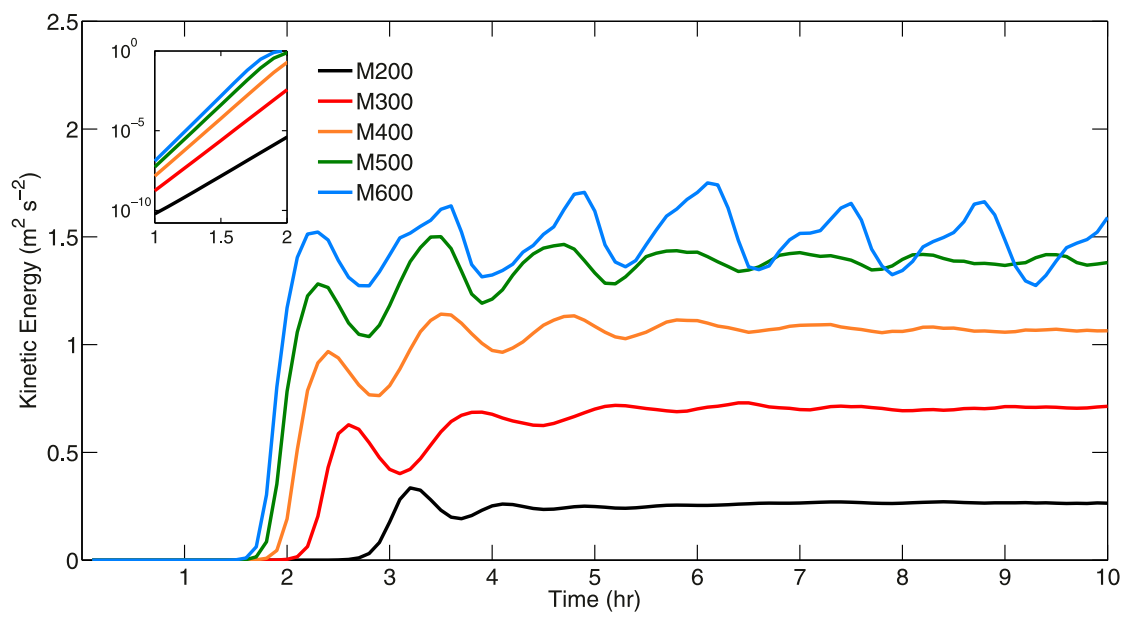

FIG. 6. Time evolution of the domain-averaged overturning kinetic energy of the perturbations in experiments M200-M600. The insert shows the kinetic energy at $1-2 \mathrm{~h}$ on a $\log$ scale.

\section{Effect of mixed-layer height on rolls and internal waves}

\section{a. Kinetic energy of the perturbations}

To describe the strength of the perturbations, we introduce the overturning kinetic energy as $e^{\prime}=$ $0.5\left(u^{\prime 2}+w^{\prime 2}\right)$. The along-roll velocity component $v^{\prime}$ is excluded from the definition of $e^{\prime}$ because $v^{\prime}$ has no direct influence on the overturning circulations $\left(u^{\prime}, w^{\prime}\right)$ in the $x-z$ plane [see (6)]. We further introduce the domain-averaged overturning kinetic energy $\left\langle\overline{e^{\prime}}\right\rangle$, where the overbar represents horizontal averaging in the cross-roll direction and $\langle\cdot\rangle$ represents vertical averaging in the lower 3-km layer of the SRM domain. The perturbations in the upper 3-km layer where the Rayleigh damping is applied will not be considered. The equation for $\left\langle\overline{e^{\prime}}\right\rangle$ are derived from the equations governing the perturbations (see appendix A) and written as

$$
\begin{aligned}
& \frac{d\left\langle\overline{e^{\prime}}\right\rangle}{d t}=\underbrace{\left\langle-\overline{w^{\prime} u^{\prime}} \frac{\partial \bar{u}}{\partial z}\right\rangle}_{S}+\underbrace{\left\langle\frac{g}{\theta_{v 0}} \overline{w^{\prime} \theta_{v}^{\prime}}\right\rangle}_{B}
\end{aligned}
$$

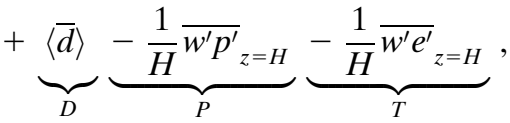

where $H=3 \mathrm{~km}$; the terms on the right-hand side are the production and loss terms for $\left\langle\overline{e^{\prime}}\right\rangle: S$ is shear production, $B$ is buoyancy work, and $D$ is turbulent dissipation, where $d=u^{\prime}\left[\partial\left(K \partial u^{\prime} / \partial x\right) / \partial x+\partial\left(K \partial u^{\prime} / \partial z\right) / \partial z\right]+$ $w^{\prime}\left[\partial\left(K \partial w^{\prime} / \partial x\right) / \partial x+\partial\left(K \partial w^{\prime} / \partial z\right) / \partial z\right] ; P$ is the loss of the kinetic energy through the pressure work $\overline{w^{\prime} p^{\prime}}$ at $z=$ $3 \mathrm{~km}$ (at the lower boundary, $\overline{w^{\prime} p^{\prime}}=0$ ); and $T$ is the loss of kinetic energy through the net vertical transport $\overline{w^{\prime} e^{\prime}}$ at $z=3 \mathrm{~km}$ (at the lower boundary, $\overline{w^{\prime} e^{\prime}}=0$ ).

Figure 6 shows the time series of $\left\langle\overline{e^{\prime}}\right\rangle$ in M200-M600. During the linear phase $\left\langle\overline{e^{\prime}}\right\rangle$ grows exponentially $\left(\left\langle\overline{e^{\prime}}\right\rangle\right.$ is nearly zero at the beginning of each experiment). Once the perturbations reach finite amplitude, they quickly reach a quasi-equilibrium state, which is defined as the period during which $\left\langle\overline{e^{\prime}}\right\rangle$ is maintained at a near-steady level. The characteristics of the perturbations in the linear phase were investigated by Gao and Ginis (2014). In this study, we focus on the perturbations during the quasi-equilibrium state. In experiments M300-M600, $\left\langle\overline{e^{\prime}}\right\rangle$ oscillates around the near-steady level in the quasiequilibrium state. The frequency of the oscillation is almost identical to the local inertial frequency $I$, corresponding to the period of $\sim 1.2 \mathrm{~h}$. This implies that the oscillation of $\left\langle\overline{e^{\prime}}\right\rangle$ originates from the inertial oscillation of the mean wind. The magnitude of $\left\langle\overline{e^{\prime}}\right\rangle$, which reflects the strength of the perturbations, increases with the mixed-layer height.

Next we investigate how the perturbations in the quasi-equilibrium state are maintained. Figure 7 shows the time series of the budget terms in (11) in M300 and M600. In both cases, shear production $S$ is the only source term for $\left\langle\overline{e^{\prime}}\right\rangle$ and it is balanced by the combination of the buoyancy work $B$, turbulent dissipation $D$, and pressure work $P$ in the quasi-equilibrium state. The vertical transport term $T$ has no net contribution (not shown). In the experiment with a higher mixed layer (M600), the shear production term is larger, suggesting that the rolls generated by the inflection-point instability can extract kinetic energy more effectively from the cross-roll mean wind. Moreover, the pressure-work term becomes more significant in balancing the shear production 

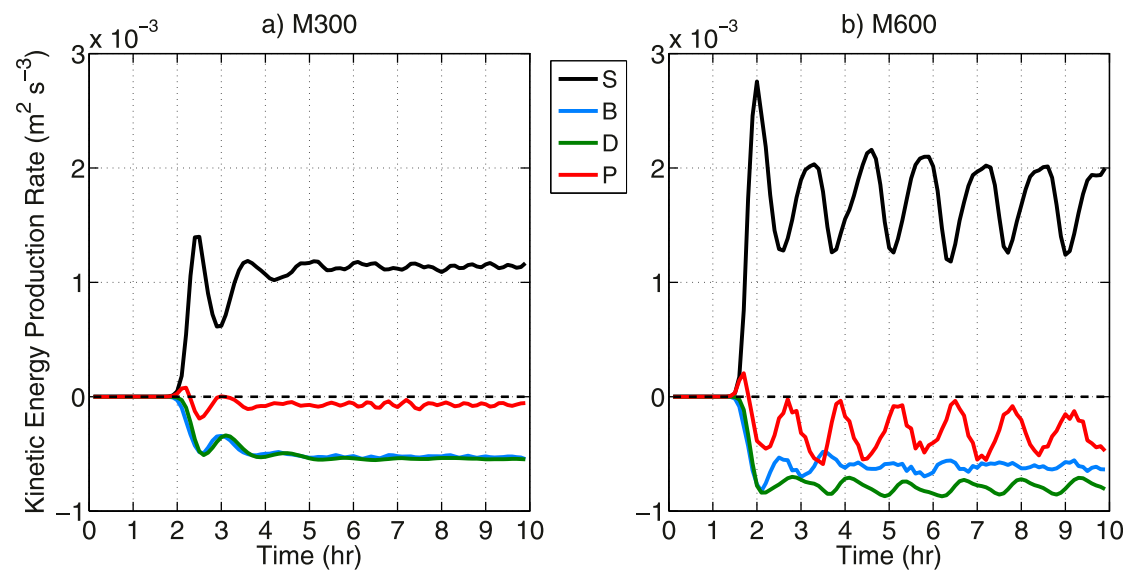

FIG. 7. Time evolution of the overturning kinetic energy budget terms in (11) in the text: $S$ is shear production, $B$ is buoyancy work, $D$ is turbulent dissipation, and $P$ is pressure work (the vertical transport term $T$ is not shown) for (a) M300 and (b) M600.

in experiment M600. In fact, $\overline{w^{\prime} p^{\prime}}$ is equivalent to the vertical energy flux transported by the internal waves at $z=3 \mathrm{~km}$. This suggests that under a higher mixed layer, the internal waves are more effectively triggered and they can carry more kinetic energy upward.

\section{b. Basic structure of the perturbations}

Figures 8 and 9 show the representative structures of perturbations in M300 and M600, respectively, in the quasi-equilibrium state. Rolls exist at the lower levels (roughly $z<1 \mathrm{~km}$ ), which have vertical velocities $w^{\prime}$ up to $\sim 3 \mathrm{~m} \mathrm{~s}^{-1}$ and cause along-roll velocity perturbations $v^{\prime}$ up to $\sim 10 \mathrm{~m} \mathrm{~s}^{-1}$. The roll horizontal wavelength, defined as the distance between two nearby $w^{\prime}$ peaks, is $\sim 3 \mathrm{~km}$. These values are within the range estimated from the radar observations (Morrison et al. 2005; Ellis and Businger 2010). The roll structures are also similar to those derived analytically by Foster (2005). Consistent with Foster's solution, the vertical velocity of rolls (Figs. 8 and 9) is asymmetric near the surface (roughly $z<0.5 \mathrm{~km}$ ): the updrafts are narrower and stronger than the downdrafts. According to Foster (1996), the asymmetry in $w^{\prime}$ is mainly due to the formation of higher-wavenumber harmonics. The spatial spectral analysis of $w^{\prime}$ in Figs. 8 and 9 reveals that the higher-wavenumber harmonics indeed exist in our numerical solutions (the analysis is not shown).
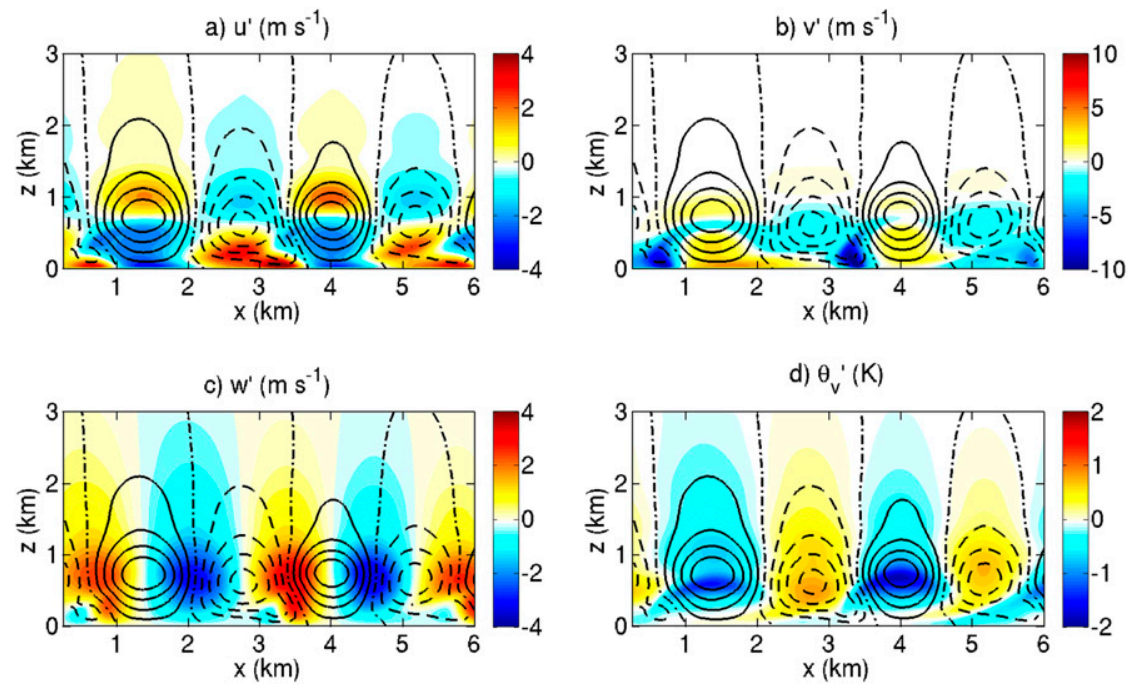

FIG. 8. Typical structure of the perturbations in M300. The colored backgrounds represent (a) cross-roll velocity $u^{\prime}$, (b) along-roll velocity $v^{\prime}$, (c) vertical velocity $w^{\prime}$, and (d) virtual potential temperature perturbation $\theta_{v}^{\prime}$. The contour lines represent the streamlines: solid (dashed) contours correspond to clockwise (counterclockwise) circulations. 

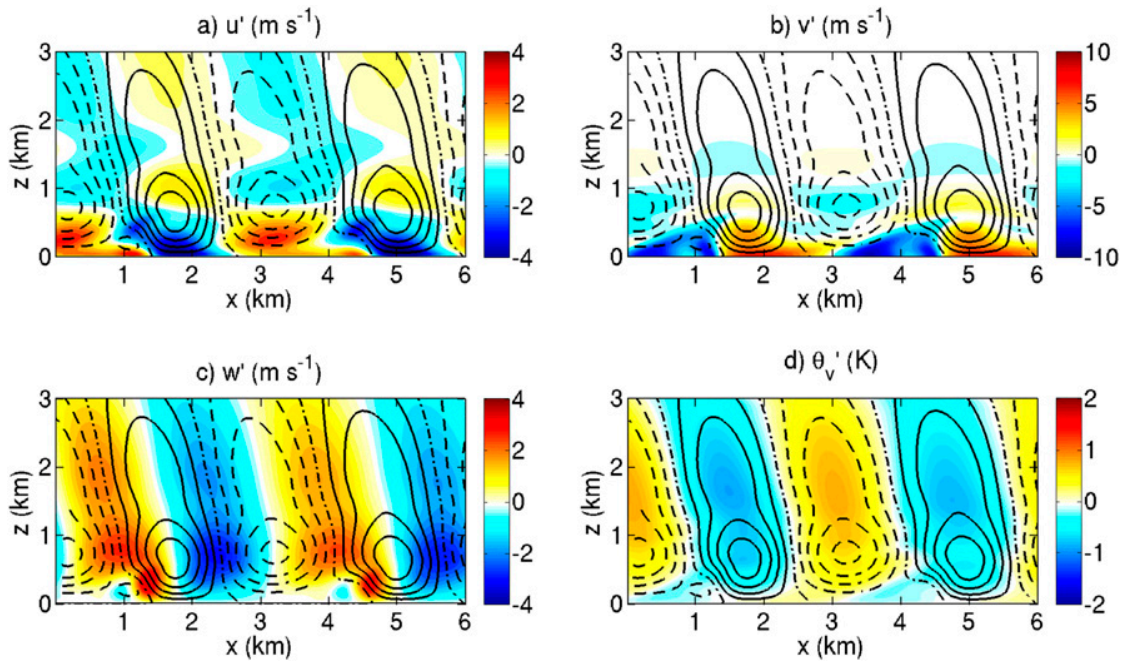

FIG. 9. As in Fig. 8, but in M600.

The most apparent differences between the perturbations in M300 and M600 (Figs. 8 and 9) are found at upper levels $(z>1 \mathrm{~km})$ : the contour lines of $\psi^{\prime}, u^{\prime}, w^{\prime}$, and $\theta_{v}^{\prime}$ are noticeably inclined from the vertical axis in M600. This is the signature of vertically propagating internal waves. As discussed by Gao and Ginis (2014), the internal waves are generated because the rolls keep perturbing the stably stratified layer above while they propagate horizontally. The internal waves are phase locked with the rolls and have the same horizontal wavenumber, horizontal phase speed, and angular frequency as the rolls. The absence of wave signature in M300 (Fig. 8) suggests that internal waves are not effectively triggered under a relatively low mixed layer, primarily because the magnitude of the overturning circulation of rolls is weaker.

\section{c. Vertical fluxes induced by the perturbations}

Figure 10 shows the cross-roll $\left(\overline{w^{\prime} u^{\prime}}\right)$ and along-roll $\left(\overline{w^{\prime} v^{\prime}}\right)$ momentum fluxes, and the virtual potential temperature flux $\overline{w^{\prime} \theta_{v}^{\prime}}$ induced by the perturbations and averaged over two inertial periods in the quasiequilibrium state. These fluxes are mostly concentrated at the lower levels $(z<1 \mathrm{~km})$ and induced by rolls in M200-M600. The momentum fluxes in different experiments have similar vertical distributions, but their magnitudes increase as the mixed-layer height increases. This is the result of stronger perturbations under higher mixed layers. The magnitude of the roll-induced momentum fluxes in the model is similar to the typical magnitude of the observed turbulent momentum fluxes reported by Zhang and Drennan (2012). At upper levels $(z>1 \mathrm{~km}), \overline{w^{\prime} u^{\prime}}$ is nonzero and vertically uniform in the experiments with relatively high mixed layers (M500 and M600), which is associated with the vertically propagating internal waves. The value of $\overline{w^{\prime} \theta_{v}^{\prime}}$ at upper levels is almost zero (Fig. 10), which is consistent with the common understanding of the internal wave transport properties. The ambient environment for the internal waves in this study is vertically uniform, and thus these waves can propagate freely without breaking. Therefore the momentum flux induced by the internal waves has no net effect on the mean flow. However, in the real atmosphere, internal waves may break via various mechanisms (Sutherland 2010), and the internal wave momentum may be deposited to the mean flow. The generation of internal waves implies that the effect of rolls in the hurricane may not be limited to the HBL.

\section{Roll-induced momentum fluxes}

We next examine the correlations between the rollinduced momentum fluxes and the mean wind shear and address the question whether the roll-induced momentum fluxes can be represented by $K$ theory commonly used in hurricane models (Kepert 2012). The $K$ theory assumes that the vertical fluxes induced by the unresolved boundary layer motions depends on the vertical gradient of the mean (or resolved) variables and can be represented as $\overline{w^{\prime} \varphi^{\prime}}=-K_{\varphi} \partial \bar{\varphi} / \partial z$, where $\varphi$ can be an arbitrary variable and $K_{\varphi}$ is the parameterized diffusivity for $\varphi$. Following Glendening (1996), we define the effective $K$ for the cross-roll momentum $K_{u}$ and alongroll momentum $K_{v}$ as follows:

$$
\begin{aligned}
& K_{u}=-\frac{\overline{w^{\prime} u^{\prime}}}{\partial \bar{u} / \partial z} \quad \text { and } \\
& K_{v}=-\frac{\overline{w^{\prime} v^{\prime}}}{\partial \bar{v} / \partial z},
\end{aligned}
$$



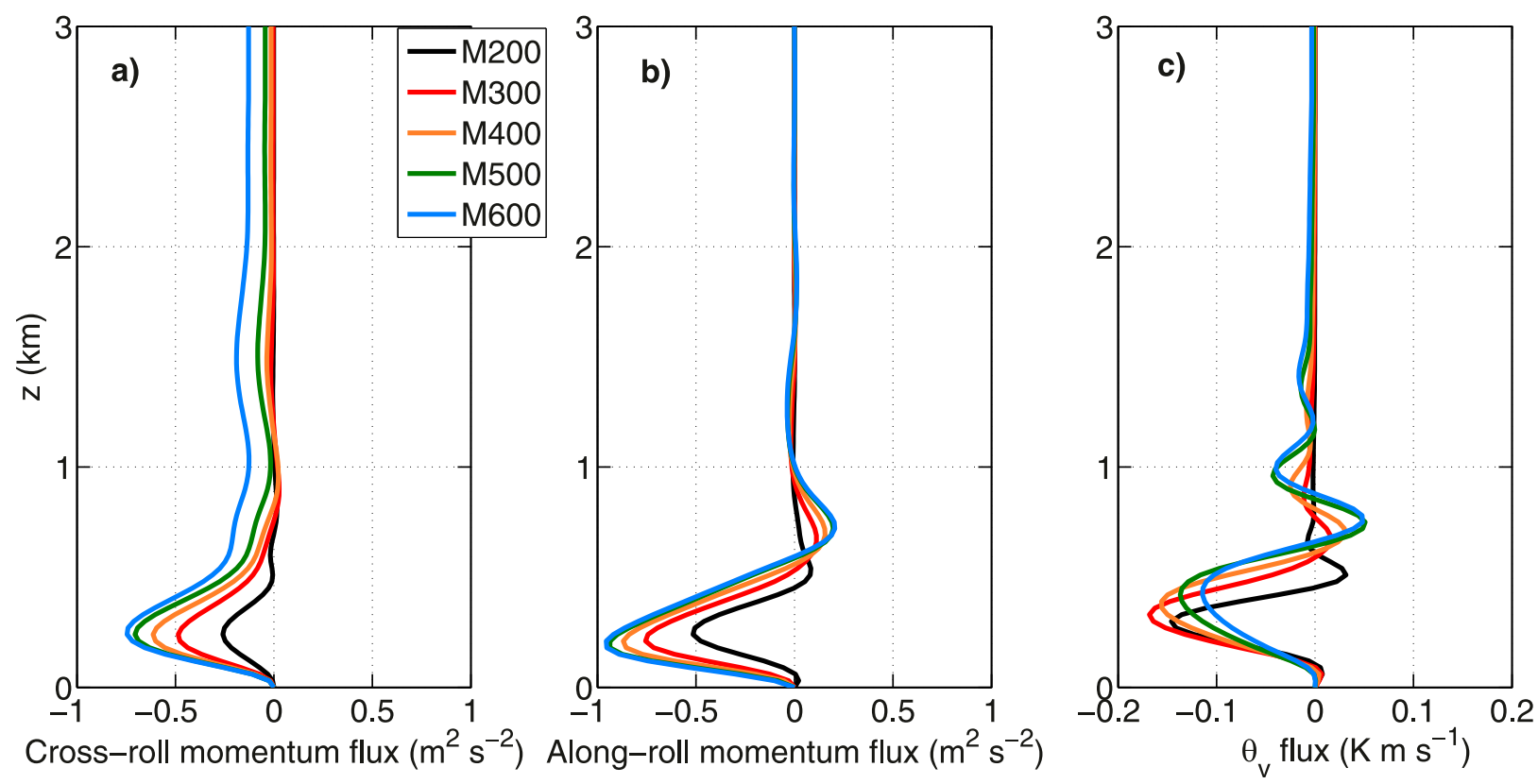

FIG. 10. Vertical profiles of the time-averaged (a) cross-roll momentum flux $\overline{w^{\prime} u^{\prime}}$, (b) along-roll momentum flux $\overline{w^{\prime} v^{\prime}}$, and (c) virtual potential temperature flux $\overline{w^{\prime} \theta_{v}^{\prime}}$ in the quasi-equilibrium state.

where the roll-induced momentum fluxes and the mean wind shear are time averaged over two inertial periods in the quasi-equilibrium state.

We find that $K_{u}$ and $K_{v}$ have very different distributions in the mid-boundary layer region $(0.1-0.6 \mathrm{~km})$, where the roll-induced fluxes are most significant (Fig. 11). In all experiments, $K_{u}$ is always positive and has finite values, while $K_{v}$ reaches infinity at $\sim 0.4 \mathrm{~km}$ and has negative values between 0.4 and $0.6 \mathrm{~km}$. To investigate this in more detail, Fig. 12 shows the profiles of the mean wind shear and the roll-induced momentum fluxes in M600. While $\overline{w^{\prime} u^{\prime}}$ is negatively correlated with $\partial \bar{u} / \partial z$, as suggested by the distribution of $K_{u}$ (Fig. 11a), it is not the case for $\overline{w^{\prime} v^{\prime}}: \partial \bar{v} / \partial z$ changes monotonically with height, but $\overline{w^{\prime} v^{\prime}}$ does not. Moreover, between 0.4 and $0.6 \mathrm{~km}$ (corresponding to the layer with negative $K_{v}$ ), $\overline{w^{\prime} v^{\prime}}$ has same sign as $\partial \bar{v} / \partial z$, which means that $\overline{w^{\prime} v^{\prime}}$ is countergradient. It should be noted that the countergradient $\overline{w^{\prime} v^{\prime}}$ is a robust feature, which does not vanish even in the case of weaker rolls (under a shallower mixed layer).

The physical explanation for the different correlations of the cross-roll and along-roll momentum fluxes with the mean wind shear is as follows. Rolls, which are
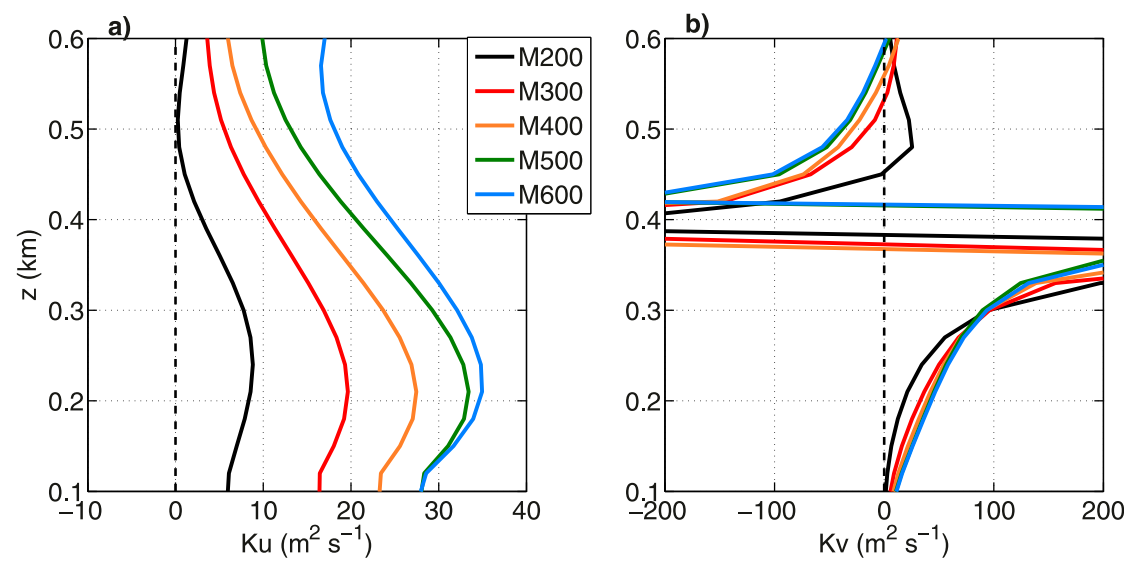

FIG. 11. Profiles of the effective $K$ calculated based on the time-averaged roll-induced momentum fluxes and the mean wind shear in the mid-boundary layer $(0.1-0.6 \mathrm{~km})$. (a) Variable $K_{u}$ is for the cross-roll momentum and (b) $K_{v}$ is for the along-roll momentum. 

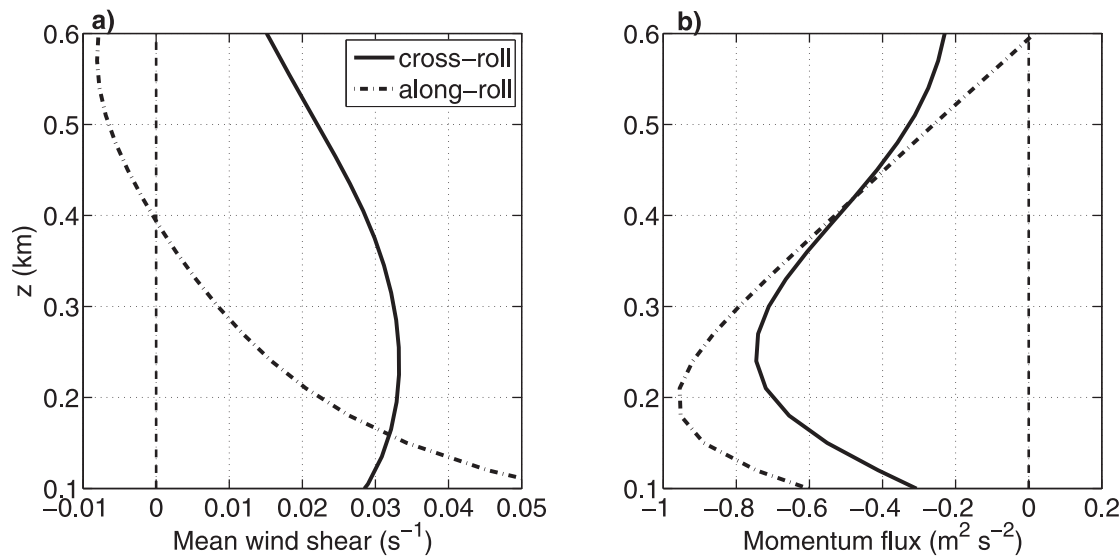

FIG. 12. Vertical profiles of the time-averaged (a) mean wind shears $(\partial \bar{u} / \partial z$ multiplied by 1.5: solid line; $\partial \bar{v} / \partial z$ : dashed line) and (b) roll-induced momentum fluxes ( $\overline{w^{\prime} u^{\prime}}$ : solid line; $\overline{w^{\prime} v^{\prime}}$ : dashed line) in the mid-boundary layer $(0.1-0.6 \mathrm{~km})$ in $\mathrm{M} 600$.

generated by the inflection point instability, gain their kinetic energy from the cross-roll mean wind $\bar{u}(z)$. In order for the shear production in (11) be positive, $\overline{w^{\prime} u^{\prime}}$ must have an opposite sign with $\partial \bar{u} / \partial z$. As discussed in Gao and Ginis (2014), the streamlines of rolls tend to tilt in such a way that $\overline{w^{\prime} u^{\prime}}$ is negatively correlated with $\partial \bar{u} / \partial z$. However, there is no such constraint on $\overline{w^{\prime} v^{\prime}}$. Physically, $\overline{w^{\prime} v^{\prime}}$ represents the net vertical transport of along-roll momentum by the overturning circulations of rolls. The along-roll mean wind $\bar{v}(z)$ (approximately the azimuthal mean wind; see Fig. 13b) is relatively slow near the surface because of the surface friction and relatively fast at higher levels. The roll updrafts move the slower-moving air parcels upward (this process creates negative $v^{\prime}$; see Fig. $8 \mathrm{~b}$ and Fig. 9b) and the roll downdrafts move the faster-moving air parcels
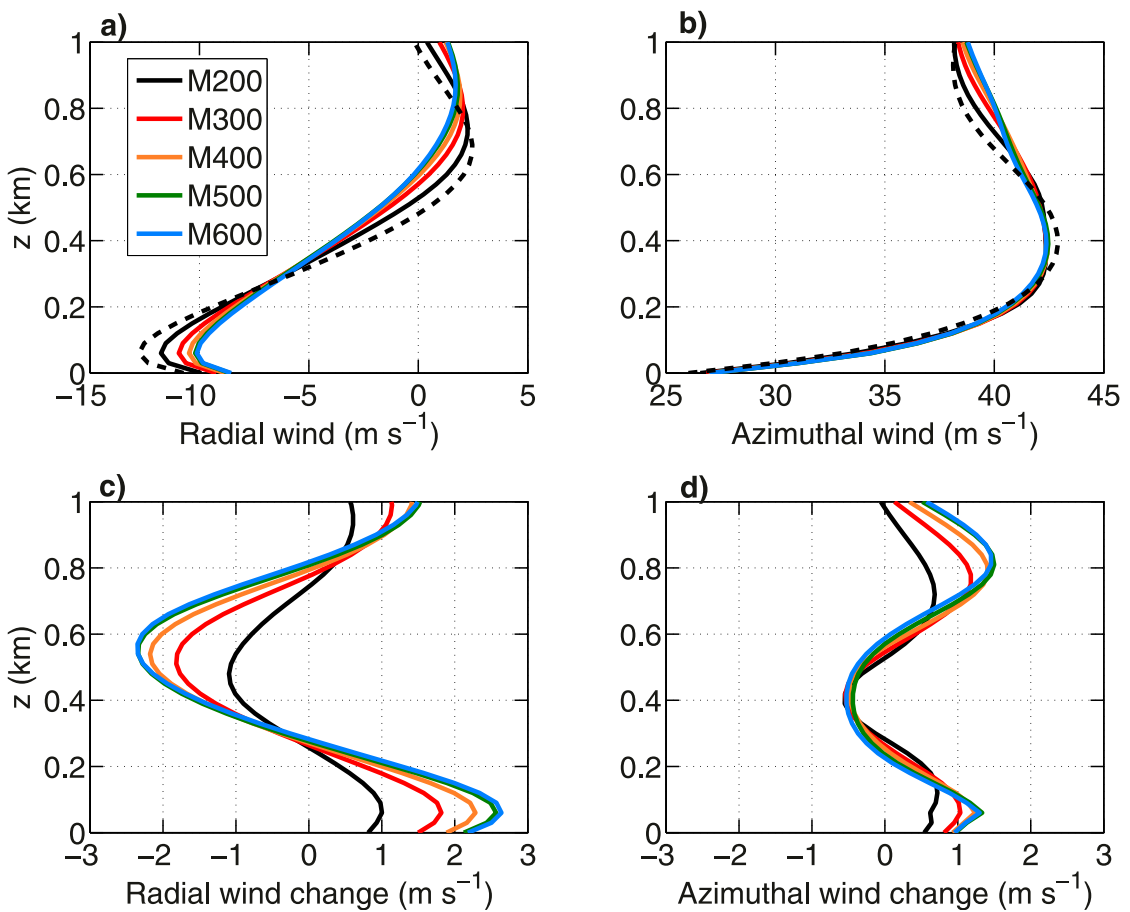

FIG. 13. The mean wind profiles with and without the effect of rolls at RMW in M200M600. (a),(b) The mean radial and azimuthal wind profiles with (without) the effect of rolls are shown as solid (dashed) lines. (c),(d) The corresponding differences of the mean wind profiles are shown. 
downward (this process creates positive $v^{\prime}$ ). As a result, $w^{\prime}$ and $v^{\prime}$ are in general negatively correlated, making $\overline{w^{\prime} v^{\prime}}$ negative throughout the vertical layer below $0.6 \mathrm{~km}$. Thus, the vertical distribution of $\overline{w^{\prime} v^{\prime}}$ is determined by a combination of the vertical motions of rolls and the overall distribution of $\bar{v}(z)$ in the boundary layer but does not necessarily correlate well with the vertical distribution of $\partial \bar{v} / \partial z$.

The above analysis suggests that the roll-induced along-roll momentum flux conflicts with the assumption of $K$ theory that the momentum fluxes are dependent on the mean shear and always downgradient. Thus, $K$ theory cannot reasonably represent the vertical distribution of the roll-induced along-roll momentum flux. It is commonly known that the large eddies in the atmospheric boundary layer can induce countergradient fluxes. To take into account the countergradient fluxes, some boundary layer parameterizations additionally consider a "nonlocal" flux component by adding a correction $r_{\varphi}$ to the vertical gradient of $\bar{\varphi}$ (Hong and Pan 1996; Hong et al. 2006). In this case, the total flux is written as $-K_{\varphi}\left(\partial \bar{\varphi} / \partial z-r_{\varphi}\right)$. The nonlocal flux component $K_{\varphi} r_{\varphi}$ is dependent on the surface flux of $\varphi$ and physically represents the contribution of the convective eddies to the total flux of $\varphi$. However, the nonlocal flux component may not be able to reasonably capture the fluxes induced by rolls in the HBL, which are driven by the shear instability but not the convective instability. The roll-induced vertical fluxes depend on the overall distribution of the mean wind and stratification in the HBL but do not directly depend on the surface fluxes. If the roll-induced fluxes are to be parameterized, we recommend developing a new scheme that is capable of capturing the characteristic distribution of the rollinduced fluxes based on the overall vertical distribution of the mean flow in the HBL.

\section{Effect of rolls on the HBL mean wind}

In this section, we discuss how rolls affect the HBL mean wind and investigate the underlying physical mechanism. The HBL model results indicate that after roll-induced momentum fluxes are introduced, the HBL mean wind profiles are adjusted to a new balanced state (corresponding to the quasi-equilibrium state of the rolls) within a few inertial cycles. We only focus on the well-adjusted mean wind, derived by averaging the mean wind profiles over two inertial cycles in the quasiequilibrium state. For convenience, we define the balanced state under the impacts of rolls as the final state and the balanced state without the impacts of rolls as the initial state. Figure 13 shows the mean wind profiles in the final and initial states at RMW in M200-M600, as well as the differences of the mean wind profiles in the two states. These differences can be considered as the net changes induced by rolls. The changes of the mean wind profiles in all experiments are qualitatively similar. Rolls in the experiments with higher mixed layers cause more significant modifications of the mean wind because they generate stronger momentum fluxes. In the radial direction (Fig. 13a), rolls weaken the inflow speed near surface (below $\sim 0.3 \mathrm{~km}$ ), enhance the inflow speed at upper levels (between $\sim 0.3$ and $\sim 0.6 \mathrm{~km}$ ), and increase the depth of the inflow layer. In the azimuthal direction (Fig. 13b), the most apparent feature is a small weakening of the supergradient jet (at $\sim 0.4 \mathrm{~km}$ ). Overall, the changes in the radial wind are more significant than those in the azimuthal wind (Figs. 13c,d). The rolls can reduce the surface inflow speed up to $\sim 2.5 \mathrm{~m} \mathrm{~s}^{-1}$ (corresponding to an $\sim 25 \%$ change) and increase the inflow layer height up to $\sim 100 \mathrm{~m}$ (corresponding to an $\sim 20 \%$ change). Such effect of rolls on the radial inflow is significant and indicates that rolls play an important role in the HBL dynamics.

To explain the impacts of rolls, we first consider the subgrid momentum tendencies in mean wind equations (2)-(3) in the initial and final states. In the HBL model, there are two types of subgrid tendencies: turbulent (parameterized) and roll induced (explicitly resolved by the SRM). Hereafter, the sum of the roll-induced and turbulent momentum tendencies or fluxes will be referred to as the total subgrid momentum tendencies or fluxes. Figure 14 shows the subgrid momentum fluxes and tendencies, which are projected onto the radial and azimuthal directions, in the initial and final states in experiment M600. Because the angle $\varepsilon$ is small, there are minor differences between the cross-roll momentum flux $\overline{w^{\prime} u^{\prime}}$ (along-roll momentum flux $\overline{w^{\prime} v^{\prime}}$ ) and the radial momentum flux $\overline{W^{\prime} U^{\prime}}$ (azimuthal momentum flux $\left.\overline{W^{\prime} V^{\prime}}\right)$.

(i) In the radial direction, the roll-induced and the turbulent momentum fluxes have very similar vertical distributions (Fig. 14a). This is mainly because both fluxes depend on the mean wind shear. While the turbulent flux is reduced in the final state, the total subgrid flux is increased as a result of the rollinduced flux. Nevertheless, the total radial momentum tendency in the final state is quite similar to that in the initial state (Fig. 14c).

(ii) In the azimuthal direction, the roll-induced and turbulent momentum fluxes have very different vertical distributions (Fig. 14b). Particularly, near the surface $(z<0.2 \mathrm{~km})$, the roll-induced flux and the turbulent flux have opposite vertical trends. As discussed in section 5 , this difference is due to the 

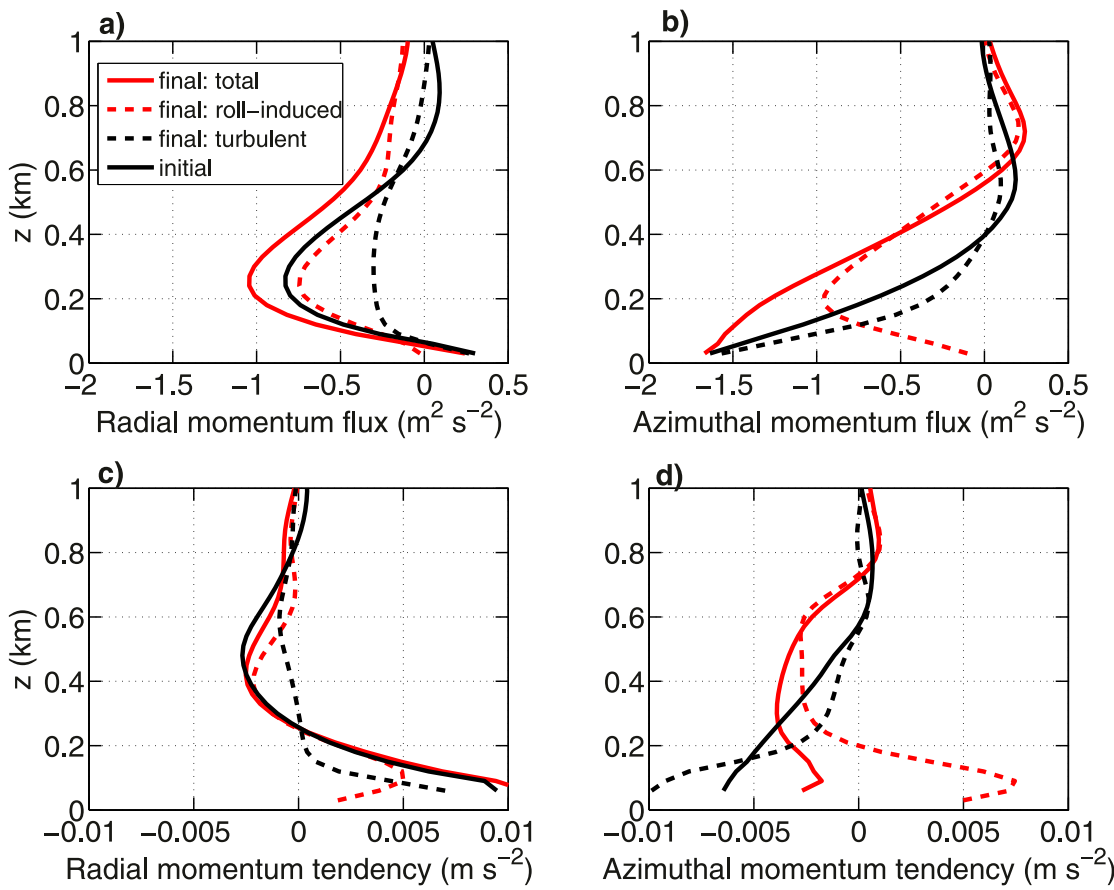

FIG. 14. The subgrid (with respect to the HBL model) momentum fluxes and tendencies in the initial and final states in M600. The initial state only has the turbulent component (black solid lines). The final state has two components: the turbulent component (black dashed lines) and the roll-induced component (red dashed lines); the total is shown by red solid lines.

fact that the roll-induced along-roll momentum flux does not depend on the local mean wind shear. The turbulent and roll-induced azimuthal momentum tendencies also have very different distributions (Fig. 14d). While the turbulent tendency is mostly negative, the roll-induced tendency is positive near the surface and negative at upper levels, and the vertically integrated roll-induced tendency is nearly zero. This indicates that the rolls cause vertical redistribution of the mean azimuthal momentum within the HBL.

To gain a physical insight into how the rolls modify the mean wind, we now consider a linear model describing the basic dynamical balances in the HBL. Following Kepert (2001), the mean wind is decomposed into two components: the gradient wind and departures from the gradient wind-that is, $\bar{V}=$ $V_{g}+V_{d}$ and $\bar{U}=U_{d}$, where $V_{g}$ is the gradient wind and $\left(U_{d}, V_{d}\right)$ are the wind departures. Based on this decomposition, linear equations governing $U_{d}$ and $V_{d}$ can be derived (Kepert 2001). Here, we denote $\left(U_{d}, V_{d}\right)$ in the initial state as $\left(U_{0}, V_{0}\right)$ and in the final state as $\left(U_{1}\right.$, $\left.V_{1}\right)$. Accordingly, the turbulent diffusivity $\bar{K}$ in the initial state is denoted as $K_{0}$ and in the final state as $K_{1}$. The linear equations for the wind departures in the initial state can be written as

$$
\begin{aligned}
-\left(f+\frac{2 V_{g}}{r}\right) V_{0} & =\frac{\partial}{\partial z}\left(K_{0} \frac{\partial U_{0}}{\partial z}\right) \text { and } \\
\left(f+\frac{V_{g}}{r}+\frac{\partial V_{g}}{\partial r}\right) U_{0} & =\frac{\partial}{\partial z}\left(K_{0} \frac{\partial V_{0}}{\partial z}\right) .
\end{aligned}
$$

The terms on the right-hand sides (RHSs) represent the turbulent momentum tendencies. According to Kepert (2001), (14) describes the balance between the radial acceleration due to the gradient wind imbalance and the turbulent radial momentum tendency and (15) describes the balance between the radial advection of the absolute angular momentum (defined as $1 / 2 f r^{2}+V_{g} r$ ) and the turbulent angular momentum dissipation. Similarly, the linear equations for the wind departures in the final state can be written as

$$
\begin{gathered}
-\left(f+\frac{2 V_{g}}{r}\right) V_{1}=\frac{\partial}{\partial z}\left(K_{1} \frac{\partial U_{1}}{\partial z}\right)-\frac{\partial \overline{W^{\prime} U^{\prime}}}{\partial z} \text { and } \\
\left(f+\frac{V_{g}}{r}+\frac{\partial V_{g}}{\partial r}\right) U_{1}=\frac{\partial}{\partial z}\left(K_{1} \frac{\partial V_{1}}{\partial z}\right)-\frac{\partial \overline{W^{\prime} V^{\prime}}}{\partial z} .
\end{gathered}
$$

Compared with (14) and (15), the additional terms on the RHS are the roll-induced momentum tendencies, which represent the effect of rolls on the radial momentum in (16) and the angular momentum in (17). By subtracting (14) and (15) from (16) and (17), we have 


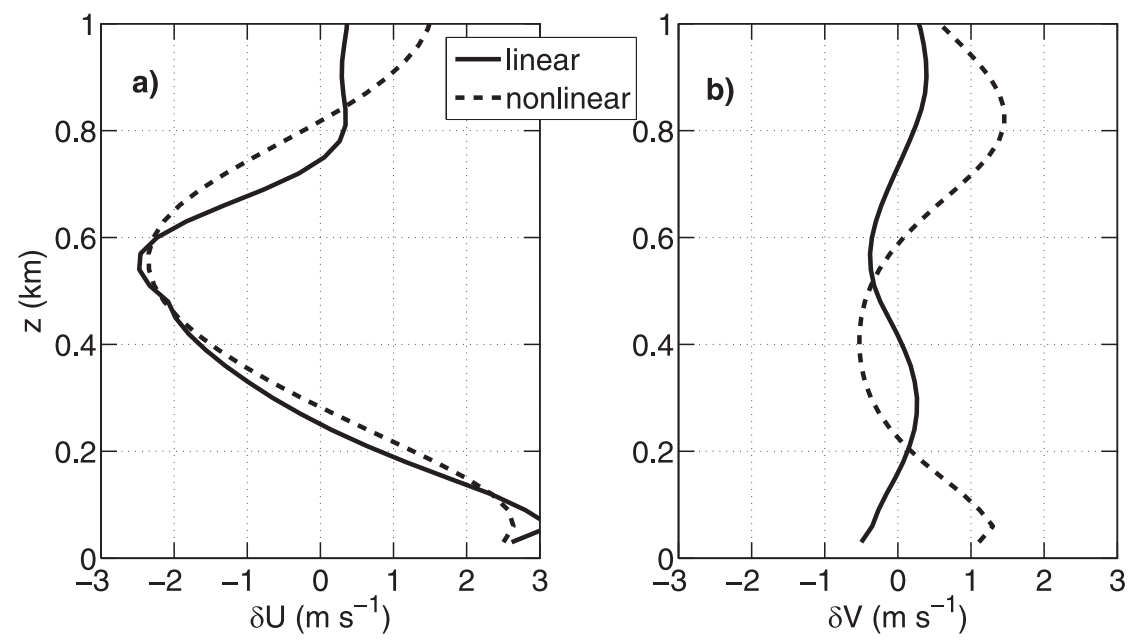

FIG. 15. The estimated mean wind changes based on the linear model (solid lines) and the actual wind changes in M600 (dashed lines).

$$
\begin{aligned}
-\left(f+\frac{2 V_{g}}{r}\right) \delta V & =\delta \mathrm{RHS}_{U} \quad \text { and } \\
\left(f+\frac{V_{g}}{r}+\frac{\partial V_{g}}{\partial r}\right) \delta U & =\delta \mathrm{RHS}_{V},
\end{aligned}
$$

where $\delta U$ and $\delta V$ represent the mean wind changes from the initial state to final state (e.g., $\delta U=U_{1}-U_{0}$ ); $\delta \mathrm{RHS}_{U}$ and $\delta \mathrm{RHS}_{V}$ represent the differences between the total subgrid tendencies in the final state and those in the initial state.

To explore whether the linear model in (18) and (19) can be used to interpret the roll-induced mean wind changes, we compare the mean wind changes $(\delta U$ and $\delta V$ ) estimated based on the linear model with those directly calculated by the HBL model (Figs. 13c,d). The values of $\delta \mathrm{RHS}_{U}$ and $\delta \mathrm{RHS}_{V}$ used in (18) and (19) are calculated from the total subgrid tendencies in the HBL model (as shown in Fig. 14), and then $\delta U$ and $\delta V$ can be estimated based on (18) and (19). Figure 15 shows the comparison of the mean wind changes estimated based on the linear model and the actual mean wind changes in M600. The estimated azimuthal wind change by the linear model, although similar in magnitude, does not match well with that in the HBL model. This is because the linear model neglects the vertical advection terms, which are important in determining the mean azimuthal wind profiles (Kepert 2001). Nevertheless, the linear model solution indicates that the radial wind change is more significant than the azimuthal wind change, which is in agreement with the HBL model, and it also captures the radial wind change reasonably well.

Since the linear model successfully captures the key features of the mean wind changes seen in the HBL model, we can use it to elucidate the physical mechanisms by which rolls affect the mean wind profiles. In the azimuthal direction, rolls redistribute the angular momentum vertically (Fig. 14d). At lower levels (below $\sim 0.3 \mathrm{~km}$ ), the turbulent angular momentum dissipation is partially balanced by the transport of angular momentum by rolls, and therefore less radial transport of the absolute angular momentum is needed; as a result, the inflow is reduced (Fig. 15a). At higher levels (roughly $0.3-0.8 \mathrm{~km}$ ), additional angular momentum loss is created as a result of the redistribution effect of rolls, which needs to be compensated by the radial transport of the absolute angular momentum; as a result, the inflow is enhanced (Fig. 15a). In the radial direction, the relatively small change of the total subgrid momentum tendency (Fig. 14c) results in the relatively small change of the azimuthal wind.

Finally, we investigate the impacts of rolls on the overall HBL mean wind structure based on the results of the additional experiment, in which SRM is embedded at all horizontal grid points (except those inside of the RMW) in the HBL model. We consider the results after a 30-h coupled HBL-SRM simulation to ensure the perturbations at all locations reach the quasiequilibrium state. The mean wind distribution under the impacts of rolls is derived by averaging the mean wind profiles during the last $10 \mathrm{~h}$. In this experiment, rolls are generated from radius $40 \mathrm{~km}$ (RMW) to radius $110 \mathrm{~km}(2.75 \mathrm{RMW})$. Rolls are not generated at larger radii because the cross-roll mean wind shear is relatively weak and the inflection point instability is suppressed by the stratification (Gao and Ginis 2014). Figure 16 shows the radius-height distribution of the mean radial and azimuthal wind changes induced by rolls. Consistent 
a) Radial wind change $\left(\mathrm{m} \mathrm{s}^{-1}\right)$

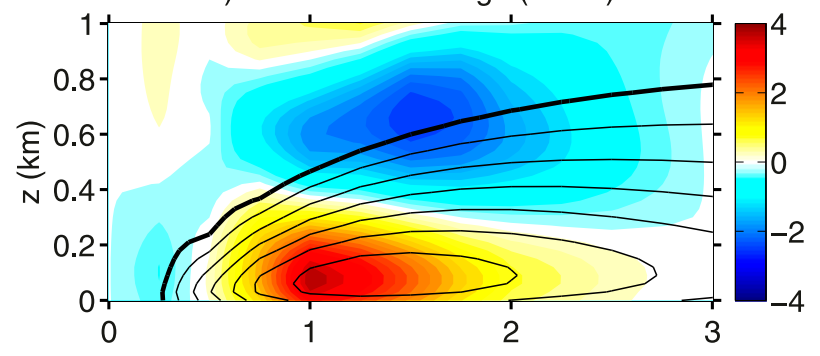

b) Azimuthal wind change $\left(\mathrm{m} \mathrm{s}^{-1}\right)$

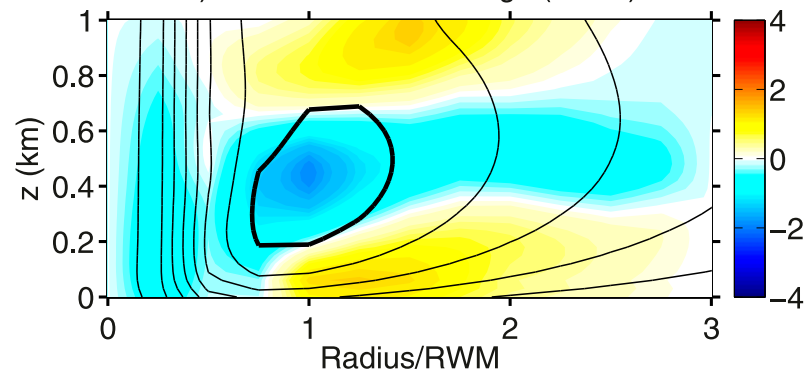

FIG. 16. Radius-height distribution of (a) radial and (b) azimuthal wind changes induced by rolls in the additional experiment. Contours represent the initial mean radial and azimuthal wind distributions (before rolls are introduced). The contour interval is (a) $2 \mathrm{~ms}^{-1}$ $\left(0 \mathrm{~m} \mathrm{~s}^{-1}\right.$ is thick) and (b) $5 \mathrm{~m} \mathrm{~s}^{-1}\left(40 \mathrm{~m} \mathrm{~s}^{-1}\right.$ is thick).

with the results of the primary experiments, rolls induce more significant changes in the radial wind. Particularly, rolls weaken the inflow near the surface and enhance the inflow at upper levels; the net effect is that the height of the inflow layer is increased.

Previous modeling studies suggest that the HBL inflow plays an important role in affecting the structure and intensity of the hurricane (e.g., Gopalakrishnan et al. 2013; Zhang et al. 2012, 2015). As indicated by our model results, the vertically averaged change of the inflow induced by rolls is small mainly because the vertically integrated roll-induced azimuthal momentum tendency is close to zero. However, the rolls introduce nonnegligible changes to the vertical profiles of the radial wind. By altering the vertical distribution of the inflow in the HBL, the rolls may affect the intensification process of the entire hurricane. In this study, the upper-hurricane vortex is fixed with time and therefore the effect of rolls on the entire hurricane cannot be explored with the use of the HBL-SRM coupled numerical system. Such investigation requires embedding the SRM into a full-physics hurricane model.

\section{Conclusions}

In this study, we applied a novel numerical approach to study rolls generated by the inflection point instability in the hurricane boundary layer. Our approach is based on embedding a two-dimensional high-resolution singlegrid roll-resolving model (SRM) at selected horizontal grid points of the axisymmetric HBL model. The SRM resolves roll motions at selected locations and provides the roll-induced momentum fluxes to the HBL model. Using this coupled HBL-SRM numerical system, we have investigated some important aspects of rolls that were not thoroughly investigated by previous studies, including the effect of the mixed-layer height on the characteristics of rolls and the internal waves triggered by rolls, the vertical distributions of the roll-induced momentum fluxes, and the effects of rolls on the HBL mean wind. The key findings are as follows.

(i) The mixed-layer height is an important environmental factor affecting the characteristics of rolls. Provided that the initial mean wind profiles are the same, rolls can reach a stronger magnitude under a higher mixed layer. Moreover, under a higher mixed layer, rolls can more efficiently trigger vertically propagating internal waves, which can radiate momentum out of the HBL.

(ii) We revealed the important difference between the roll-induced cross-roll (nearly radial) and alongroll (nearly azimuthal) momentum fluxes: while the roll-induced cross-roll momentum flux is well correlated with cross-roll mean wind shear, the rollinduced along-roll momentum flux is typically not correlated with the along-roll mean wind shear. Therefore $K$ theory commonly used in the boundary layer parameterizations cannot reasonably represent the roll-induced along-roll momentum flux.

(iii) We found that rolls can induce more significant changes in the mean radial wind than in the mean azimuthal wind. In particular, rolls reduce the inflow near surface, increase the inflow at higher levels, and broaden the inflow layer. Based on a linear dynamical HBL model, we found that the impacts of rolls on the mean radial wind distribution are essentially because of their vertical redistribution effect on the angular momentum (or the azimuthal momentum) in the HBL.

In summary, we investigated several key aspects of the boundary layer rolls in hurricanes. We offered physical explanations for why the roll-induced vertical momentum fluxes cannot be properly represented by the commonly used parameterizations in hurricane models. We also suggest that the boundary layer rolls may contribute to the intensification process of the entire hurricane. Future work will investigate possible physical mechanisms of how boundary layer rolls may contribute to the hurricane intensity change.

Acknowledgments. We would like to acknowledge Ralph Foster for his valuable input throughout this study 
and Jun Zhang for providing the composite hurricane boundary layer observations. We also want to thank Jun Zhang and two anonymous reviewers for providing the insightful comments that significantly improved this paper. This research was funded by the Office of Navy Research through Award N000141210447.

\section{APPENDIX A}

\section{Overturning Kinetic Energy Budget Equation for the Perturbations}

Here we show the derivations of the overturning kinetic energy budget equation for the perturbations. Without introducing the streamfunction and the alongroll vorticity, the equations governing the overturning circulation $\left(u^{\prime}, w^{\prime}\right)$ of the perturbations are

$$
\begin{aligned}
& \frac{\partial u^{\prime}}{\partial t}+u^{\prime} \frac{\partial u^{\prime}}{\partial x}+w^{\prime} \frac{\partial u^{\prime}}{\partial z}=-\bar{u} \frac{\partial u^{\prime}}{\partial x}-w^{\prime} \frac{\partial \bar{u}}{\partial z}-\frac{\partial p^{\prime}}{\partial x}+T_{u^{\prime}}, \quad \text { (A1) } \\
& \frac{\partial w^{\prime}}{\partial t}+u^{\prime} \frac{\partial w^{\prime}}{\partial x}+w^{\prime} \frac{\partial w^{\prime}}{\partial z}=-\bar{u} \frac{\partial w^{\prime}}{\partial x}-\frac{\partial p^{\prime}}{\partial z}+\frac{g}{\theta_{v 0}} \theta_{v}^{\prime}+T_{w^{\prime}}, \quad \text { and }
\end{aligned}
$$

$$
\frac{\partial u^{\prime}}{\partial x}+\frac{\partial w^{\prime}}{\partial z}=0
$$

where $T_{u^{\prime}}$ and $T_{w^{\prime}}$ represent the turbulent diffusions. Take $T_{u^{\prime}}$ for example: it is in the form of $T_{u^{\prime}}=$ $\partial\left(K \partial u^{\prime} / \partial x\right) / \partial x+\partial\left(K \partial w^{\prime} / \partial z\right) / \partial z$, where $K$ is the parameterized turbulent diffusivity. Equations (A1)-(A3) are equivalent to (6) and (7) in the main text.

By combining (A1) multiplied by $u^{\prime}$ and (A2) multiplied by $w^{\prime}$, and considering (A3), we can derive the governing equation for the overturning kinetic energy $e^{\prime}$, which is given by

$$
\begin{aligned}
\frac{\partial e^{\prime}}{\partial t}+\frac{\partial u^{\prime} e^{\prime}}{\partial x}+\frac{\partial w^{\prime} e^{\prime}}{\partial z}= & -\frac{\partial \bar{u} e^{\prime}}{\partial x}-\frac{\partial u^{\prime} p^{\prime}}{\partial x}-\frac{\partial w^{\prime} p^{\prime}}{\partial z} \\
& -w^{\prime} u^{\prime} \frac{\partial \bar{u}}{\partial z}+\frac{g}{\theta_{v 0}} w^{\prime} \theta_{v}^{\prime}+d,
\end{aligned}
$$

where

$$
e^{\prime}=\frac{1}{2}\left(u^{\prime 2}+w^{\prime 2}\right)
$$

and

$$
\begin{aligned}
d= & \frac{\partial}{\partial x}\left(K \frac{\partial u^{\prime}}{\partial x}\right) u^{\prime}+\frac{\partial}{\partial z}\left(K \frac{\partial u^{\prime}}{\partial z}\right) u^{\prime}+\frac{\partial}{\partial x}\left(K \frac{\partial w^{\prime}}{\partial x}\right) w^{\prime} \\
& +\frac{\partial}{\partial z}\left(K \frac{\partial w^{\prime}}{\partial z}\right) w^{\prime} .
\end{aligned}
$$

By averaging (A4) in the $x$ (cross roll) direction, we can rule out the terms with the $x$ derivative and get

$\frac{\partial \overline{e^{\prime}}}{\partial t}+\frac{\partial \overline{w^{\prime} e^{\prime}}}{\partial z}=-\frac{\partial \overline{w^{\prime} p^{\prime}}}{\partial z}-\overline{w^{\prime} u^{\prime}} \frac{\partial \bar{u}}{\partial z}+\frac{g}{\theta_{v 0}} \overline{w^{\prime} \theta_{v}^{\prime}}+\bar{d}$

In this study, the additional Rayleigh damping is applied to damp out the internal waves above $z=3 \mathrm{~km}$, and therefore the perturbations in these levels will not be considered. By averaging (A5) vertically over the range $0-3 \mathrm{~km}$, and considering $\overline{w^{\prime} p^{\prime}}{ }_{z=0}=\overline{w^{\prime} e^{\prime}}{ }_{z=0}=0$, we eventually get

$$
\begin{aligned}
\frac{d\left\langle\overline{e^{\prime}}\right\rangle}{d t}= & \left\langle-\overline{w^{\prime} u^{\prime}} \frac{\partial \bar{u}}{\partial z}\right\rangle+\left\langle\frac{g}{\theta_{v 0}} \overline{w^{\prime} \theta_{v}^{\prime}}\right\rangle+\langle\bar{d}\rangle \\
& -\frac{1}{H} \overline{w^{\prime} p^{\prime}}{ }_{z=H}-\frac{1}{H} \overline{w^{\prime} e^{\prime}}{ }_{z=H},
\end{aligned}
$$

where $H=3 \mathrm{~km}$.

\section{APPENDIX B}

\section{Sensitivity of the Roll-Induced Momentum Fluxes and Their Impacts to Angle $\varepsilon$}

Because the SRM is two dimensional, the orientation of its domain needs to be specified when it is embedded into the mean-flow model. The traditional stability analysis sweeps through the wavenumber and angle space (e.g., Foster 2005) to find out the optimal angle at which the rolls have the largest growth rate. However, this method is not practical for the numerical modeling approach applied in this study. In the experiments presented above, the two-dimensional SRM domain is assumed perpendicular to the vertically averaged wind vector below $z=1 \mathrm{~km}$. Such method produces a small angle $\varepsilon$ (as in Fig. 1) slightly to the left of the azimuthal direction, which is consistent with Foster (2005).

To explore how the choice of $\varepsilon$ affects the roll-induced fluxes and their impacts on the mean wind profiles, we performed a few sensitivity experiments in which $\varepsilon$ in the experiments described in the main text is decreased or increased up to $100 \%$. The results of these sensitivity experiments are consistent and we only present the results from a representative group of experiments here (Fig. B1). These experiments are M600 with $\varepsilon$ set to $\sim 4^{\circ}$, M600-a with $\varepsilon$ set to $0^{\circ}$ (decreased by $100 \%$ ), and M600b with $\varepsilon$ set to $8^{\circ}$ (increased by $100 \%$ ). The vertical distributions of the roll-induced momentum fluxes (Figs. B1a,b) are not significantly affected by the variation of $\varepsilon$ and only their magnitudes are slightly affected (the change is $10 \%$ at most). The magnitudes of the rollinduced cross-roll and along-roll momentum fluxes in 

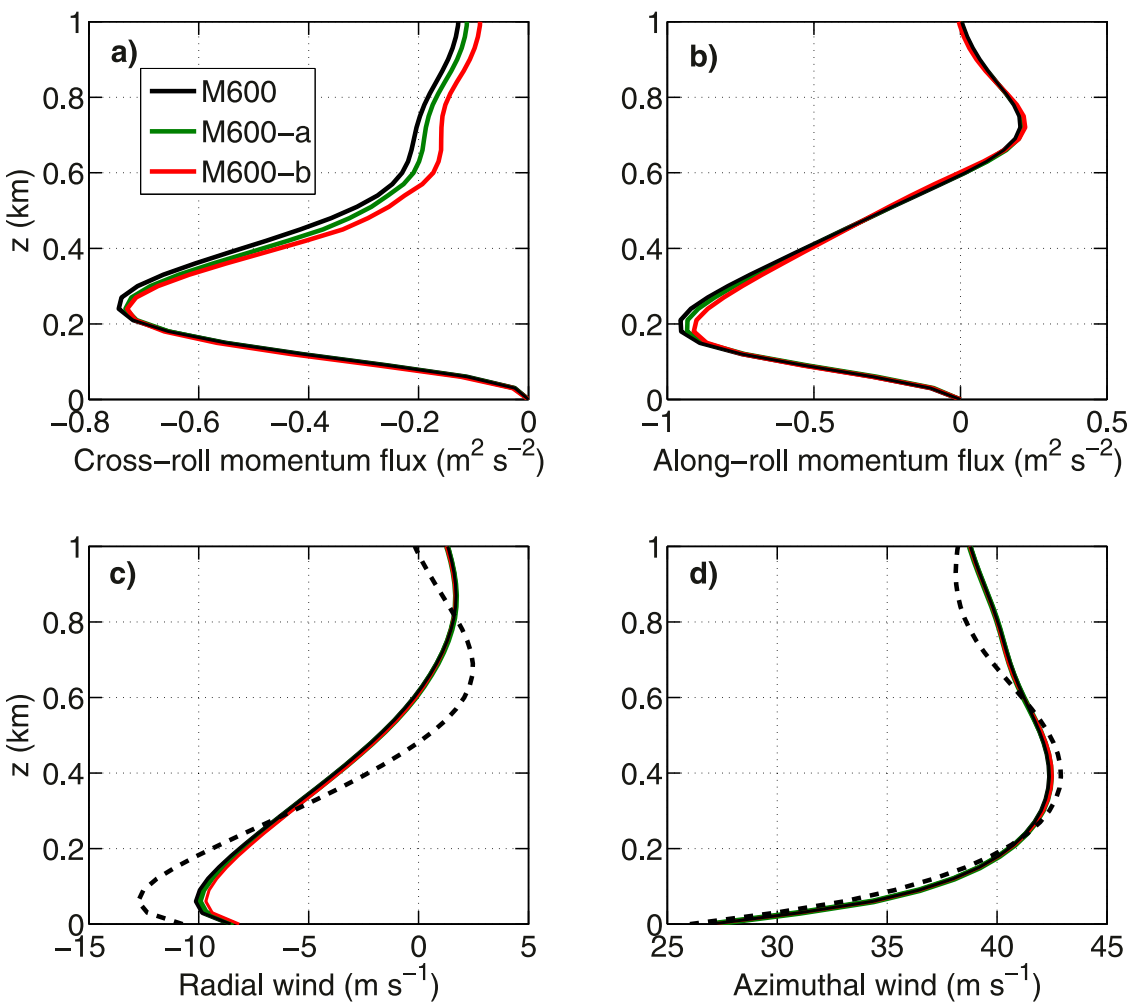

FIG. B1. Vertical profiles of the time-averaged (a) cross-roll momentum flux, (b) along-roll momentum flux, (c) radial wind, and (d) azimuthal wind in the sensitivity experiments. The variable $\varepsilon$ is reduced by $100 \%$ in M600-a and increased by $100 \%$ in M600-b. Dashed lines in (c) and (d) are the initial mean wind profiles before rolls are introduced.

both M600-a and M600-b are reduced as compared to those in M600, suggesting the choice of $\varepsilon$ in M600 is more likely to capture the most energetic rolls. The mean wind profiles (Figs. B1c,d) are hardly affected by the variation of $\varepsilon$, which is mainly because the changes in the roll-induced momentum fluxes with $\varepsilon$ are small. In summary, the sensitivity experiments suggest that the results presented in the main text are robust and not sensitive to the choice of $\varepsilon$.

\section{REFERENCES}

Blackadar, A. K., 1962: The vertical distribution of wind and turbulent exchange in a neutral atmosphere. J. Geophys. Res., 67, 3095-3102, doi:10.1029/JZ067i008p03095.

Brown, R. A., 1970: A secondary flow model of the planetary boundary layer. J. Atmos. Sci., 27, 742-757, doi:10.1175/ 1520-0469(1970)027<0742:ASFMFT>2.0.CO;2.

_ 1972: On the inflection point instability of a stratified Ekman boundary layer. J. Atmos. Sci., 29, 850-859, doi:10.1175/ 1520-0469(1972)029<0850:OTIPIO > 2.0.CO;2.

- 1980: Longitudinal instability and secondary flows in the planetary boundary layer: A review. Rev. Geophys., 18, 683697, doi:10.1029/RG018i003p00683.

Ellis, R., and S. Businger, 2010: Helical circulation in the typhoon boundary layer. J. Geophys. Res., 115, D06205, doi:10.1029/ 2009JD011819.
Etling, D., and S. Raasch, 1987: Numerical simulation of vortex roll development during a cold air outbreak. Dyn. Atmos. Oceans, 10, 277-290, doi:10.1016/0377-0265(87)90021-2.

— , and R. A. Brown, 1993: Roll vortices in the planetary boundary layer: A review. Bound.-Layer Meteor., 65, 215-248, doi:10.1007/BF00705527.

Faller, A. J., 1965: Large eddies in the atmospheric boundary layer and their possible role in the formation of cloud rows. J. Atmos. Sci., 22, 176-184, doi:10.1175/1520-0469(1965)022<0176: LEITAB $>2.0 . \mathrm{CO} ; 2$.

Foster, R., 1996: An analytic model for planetary boundary roll vortices. Ph.D. dissertation, University of Washington, 193 pp. 2005: Why rolls are prevalent in the hurricane boundary layer. J. Atmos. Sci., 62, 2647-2661, doi:10.1175/JAS3475.1. 2009: Boundary-layer similarity under an axisymmetric, gradient wind vortex. Bound.-Layer Meteor., 131, 321-344, doi:10.1007/s10546-009-9379-1.

, 2013: Signature of large aspect ratio roll vortices in synthetic aperture radar images of tropical cyclones. Oceanography, 26 (2), 58-67, doi:10.5670/oceanog.2013.31.

Gao, K., and I. Ginis, 2014: On the generation of roll vortices due to the inflection point instability of the hurricane boundary layer flow. J. Atmos. Sci., 71, 4292-4307, doi:10.1175/JAS-D-13-0362.1.

Glendening, J. W., 1996: Lineal eddy features under strong shear conditions. J. Atmos. Sci., 53, 3430-3449, doi:10.1175/ 1520-0469(1996)053<3430:LEFUSS $>2.0$. CO;2.

Gopalakrishnan, S. G., F. Marks, J. A. Zhang, X. Zhang, J.-W. Bao, and V. Tallapragada, 2013: A study of the impacts of vertical 
diffusion on the structure and intensity of tropical cyclones using the high-resolution HWRF system. J. Atmos. Sci., 70, 524-541, doi:10.1175/JAS-D-11-0340.1.

Holland, G. J., 1980: An analytic model of the wind and pressure profiles in hurricanes. Mon. Wea. Rev., 108, 1212-1218, doi:10.1175/1520-0493(1980)108<1212:AAMOTW>2.0.CO;2.

Hong, S.-Y., and H.-L. Pan, 1996: Nonlocal boundary layer vertical diffusion in a medium-range forecast model. Mon. Wea. Rev., 124, 2322-2339, doi:10.1175/1520-0493(1996)124<2322: NBLVDI $>2.0 . \mathrm{CO} ; 2$.

, Y. Noh, and J. Dudhia, 2006: A new vertical diffusion package with an explicit treatment of entrainment processes. Mon. Wea. Rev., 134, 2318-2341, doi:10.1175/MWR3199.1.

Kepert, J. D., 2001: The dynamics of boundary layer jets within the tropical cyclone core. Part I: Linear theory. J. Atmos. Sci., 58, 2469-2484, doi:10.1175/1520-0469(2001)058<2469: TDOBLJ $>2.0 . \mathrm{CO} ; 2$.

— 2012: Choosing a boundary layer parameterization for tropical cyclone modeling. Mon. Wea. Rev., 140, 1427-1445, doi:10.1175/MWR-D-11-00217.1.

— within the tropical cyclone core. Part II: Nonlinear enhancement. J. Atmos. Sci., 58, 2485-2501, doi:10.1175/ 1520-0469(2001)058<2485:TDOBLJ $>2.0$. CO $; 2$.

Lilly, D., 1966: On the instability of Ekman boundary flow. J. Atmos. Sci. 23, 481-494, doi:10.1175/1520-0469(1966)023<0481: OTIOEB $>2.0 . \mathrm{CO} ; 2$.

Lorsolo, S., J. L. Schroeder, P. Dodge, and F. Marks, 2008: An observational study of hurricane boundary layer small-scale coherent structures. Mon. Wea. Rev., 136, 2871-2893, doi:10.1175/2008MWR2273.1.

Moon, I.-J., I. Ginis, T. Hara, and B. Thomas, 2007: A physicsbased parameterization of air-sea momentum flux at high wind speeds and its impact on hurricane intensity predictions. Mon. Wea. Rev., 135, 2869-2878, doi:10.1175/MWR3432.1.

Morrison, I., S. Businger, F. Marks, P. Dodge, and J. Businger, 2005: An observational case for the prevalence of roll vortices in the hurricane boundary layer. J. Atmos. Sci., 62, 2662-2673, doi:10.1175/JAS3508.1.

Nakanishi, M., and H. Niino, 2012: Large-eddy simulation of roll vortices in a hurricane boundary layer. J. Atmos. Sci., 69, 35583575, doi:10.1175/JAS-D-11-0237.1.

Nolan, D. S., 2005: Instabilities in hurricane-like boundary layers. Dyn. Atmos. Oceans, 40, 209-236, doi:10.1016/ j.dynatmoce.2005.03.002.

Sutherland, B. R., 2010: Internal Gravity Waves. Cambridge University Press, 378 pp.

Wurman, J., and J. Winslow, 1998: Intense sub-kilometer-scale boundary layer rolls in Hurricane Fran. Science, 280, 555-557, doi:10.1126/science.280.5363.555.

Zhang, J. A., and W. M. Drennan, 2012: An observational study of vertical eddy diffusivity in the hurricane boundary layer. J. Atmos. Sci., 69, 3223-3236, doi:10.1175/JAS-D-11-0348.1.

_ K. B. Katsaros, P. G. Black, S. Lehner, J. R. French, and W. M. Drennan, 2008: Effects of roll vortices on turbulent fluxes in the hurricane boundary layer. Bound.-Layer Meteor., 128, 173-189, doi:10.1007/s10546-008-9281-2.

_, R. F. Rogers, D. S. Nolan, and F. D. Marks Jr., 2011: On the characteristic height scales of the hurricane boundary layer. Mon. Wea. Rev., 139, 2523-2535, doi:10.1175/ MWR-D-10-05017.1.

—, S. Gopalakrishnan, F. D. Marks, R. F. Rogers, and V. Tallapragada, 2012: A developmental framework for improving hurricane model physical parameterization using aircraft observations. Trop. Cyclone Res. Rev., 1, 419-429, doi:10.6057/2012tcrr04.01.

— D. S. Nolan, R. F. Rogers, and V. Tallapragada, 2015: Evaluating the impact of improvements in the boundary layer parameterization on hurricane intensity and structure forecasts in HWRF. Mon. Wea. Rev., 143, 3136-3155, doi:10.1175/ MWR-D-14-00339.1.

Zhu, P., 2008: Simulation and parameterization of the turbulent transport in the hurricane boundary layer by large eddies. J. Geophys. Res., 113, D17104, doi:10.1029/2007JD009643. 
Copyright of Journal of the Atmospheric Sciences is the property of American Meteorological Society and its content may not be copied or emailed to multiple sites or posted to a listserv without the copyright holder's express written permission. However, users may print, download, or email articles for individual use. 